\title{
A New Scale-Invariant Lindley Extension Distribution and Its Applications
}

\author{
Mohamed Kayid (D), Rayof Alskhabrah $(\mathbb{D}$, and Arwa M. Alshangiti $i$ \\ Department of Statistics and Operations Research, College of Sciences, King Saud University, Riyadh, Saudi Arabia \\ Correspondence should be addressed to Mohamed Kayid; drkayid@ksu.edu.sa
}

Received 27 June 2021; Revised 13 August 2021; Accepted 18 August 2021; Published 31 August 2021

Academic Editor: Sanku Dey

Copyright (C) 2021 Mohamed Kayid et al. This is an open access article distributed under the Creative Commons Attribution License, which permits unrestricted use, distribution, and reproduction in any medium, provided the original work is properly cited.

\begin{abstract}
A new scale-invariant extension of the Lindley distribution and its power generalization has been introduced. The moments and the moment-generating functions of the proposed models have closed forms. The failure rate, the mean residual life, and the $\alpha$-quantile residual life functions have been explored. The failure rate function of these models accommodates increasing, bathtubshaped, and increasing then bathtub-shaped forms. The parameters of the models have been estimated by the maximum likelihood method for the complete and right-censored data. In a simulation study, the efficiency and consistency of the maximum likelihood estimator have been investigated. Then, the proposed models were fitted to four data sets to show their flexibility and applicability.
\end{abstract}

\section{Introduction}

The Lindley distribution introduced by Lindley [1] has been attracted much interest in recent years. The probability density function (PDF) and the cumulative distribution function (CDF) of the Lindley are, respectively, as follows:

$$
\begin{aligned}
& f(x)=\frac{\theta^{2}}{1+\theta}(1+x) e^{-\theta x}, \quad \theta>0, x \geq 0, \\
& F(x)=1-\frac{1+\theta+\theta x}{1+\theta} e^{-\theta x}, \quad \theta>0, x \geq 0 .
\end{aligned}
$$

Clearly the Lindley distribution is a mixture of two gamma distributions $G(1, \theta)$, gamma with shape parameter 1 and scale $\theta$ and $G(2, \theta)$ with weights $\theta / 1+\theta$ and $1 / 1+\theta$, respectively.

Ghitany et al. [2] explored some properties of the Lindley model. Many authors introduced generalizations of this model or applied it in their models. Among them, Shanker and Mishra [3], Shanker and Ghebretsadik [4], Merovci and Sharma [5], Zakerzade and Dolati [6], Ibrahim et al. [7], and Shanker et al. [8] introduced generalizations of Lindley with some more parameters. Moreover, Sankaran [9], Zamani and Ismail [10], Ghitany et al. [11], Al-Mutairi et al. [12], Al-
Babtain et al. [13], Ghitany et al. [14], Al-Mutairi et al. [15], Abouammoh et al. [16], Ibrahim et al. [17], Marthin and Rao [18], Al-Babtain et al. [19], Joshi and Kumar [20], Afify et al. [21], Chesneau et al. [22], Algarni [23], and many others used the Lindley distribution in their research or extended it. These generalized models are used in the reliability theory and are useful for describing data from different sources, e.g., lifetime of an object, strength of a material, and so on.

One distribution family $f(x)$ is said to be scale invariant if $f(x)$ times the transformation Jacobian remains unchanged after transforming $x$ to $\alpha x$ (scale transform). So, changing the scale or units of $x$ leaves the fit invariant. Shanker and Mishra [3] proposed one scale-invariant quasi Lindley distribution. Unfortunately, the Lindley model and some of its generalizations do not satisfy this property. The aim of this paper is to introduce one scale invariant extension of the Lindley distribution.

In Sections 2 and 3, the new distributions are defined, and some of their properties have been studied. In Section 4, the estimation of the parameters has been discussed. Section 5 is devoted to investigation of the maximum likelihood estimator (MLE) of the parameters and its behaviour by simulation. In Section 6, four data sets have been analyzed by the proposed distributions to show their applicability. 


\section{New Lindley Extension}

We introduce one extended Lindley (EL) distribution with parameters $k>0, m>0, k \neq m, \delta>0$, and $\theta>0$, namely, $\operatorname{EL}(k, m ; \delta, \theta)$, with the PDF as follows:

$$
f(x)=\frac{\theta}{\Gamma(m)+\delta}\left(\delta \frac{(\theta x)^{k-1}}{\Gamma(k)}+(\theta x)^{m-1}\right) e^{-\theta x}, \quad x \geq 0 .
$$

It is a mixture of two gamma distributions $G(m, \theta)$ and $G(k, \theta)$ with weights $\Gamma(m) / \Gamma(m)+\delta$ and $\delta / \Gamma(m)+\delta$, respectively. In applications, when we want to fit this model to one data set, we may consider $k$ and $m$ as integers (discrete), e.g., $k, m=1,2, \ldots$.. So, we can consider a suitable sequence of pairs $(k, m)$ and search for a good model by estimating $(\delta, \theta)$. In this way, we avoid headaches of optimizing four dimensional functions.

For $m=2, k=1$, and $\delta=\theta$, it is the Lindley model. Figure 1 shows the PDF for some various parameters and indicates that the PDF accommodates decreasing, unimodal, and biomodal forms.

It is straightforward to show that the CDF of $\mathrm{EL}(k, m ; \delta, \theta)$ is

$$
F(x)=1-\frac{1}{\Gamma(m)+\delta}\left(\frac{\delta}{\Gamma(k)} \Gamma(\theta x, k)+\Gamma(\theta x, m)\right), \quad x \geq 0,
$$

in which $\Gamma(x, \alpha)=\int_{x}^{\infty} t^{\alpha-1} e^{-t} \mathrm{~d} t$ is the upper incomplete gamma function. Let $X \sim \operatorname{EL}(k, m ; \delta, \theta)$, then the $r^{\text {th }}$ moment is

$$
E\left(X^{r}\right)=\frac{1}{\theta^{r}} \frac{1}{\Gamma(m)+\delta}\left(\delta \frac{\Gamma(k+r)}{\Gamma(k)}+\Gamma(m+r)\right) .
$$

It is clear that when $k>1$ and $m>1, E\left(X^{r}\right)>1 / \theta^{r}$. The moment-generating function can also be simplified to

$$
E\left(e^{t X}\right)=\frac{1}{\Gamma(m)+\delta}\left(\left(\frac{\theta}{\theta-t}\right)^{m} \Gamma(m)+\delta\left(\frac{\theta}{\theta-t}\right)^{k}\right) .
$$

2.1. Dynamic Measures. One of the most elementary measures in reliability theory and survival analysis is the reliability function, $R(x)=1-F(x)$. Moreover, dynamic measures like the failure rate function, the mean residual life (MRL) function, and the $\alpha$-quantile residual life ( $\alpha$-QRL) function play key role in investigation the behaviour of lifetime. Let $X \sim \operatorname{EL}(k, m ; \delta, \theta)$, then the failure rate function is

$$
h(x)=\frac{f(x)}{R(x)}=\frac{\Gamma(k)(\theta x)^{m-1}+\delta(\theta x)^{k-1}}{\Gamma(k) \Gamma(\theta x, m)+\delta \Gamma(\theta x, k)} \theta e^{-\theta x} .
$$

Applying Glaser's technique, this failure rate function may exhibit increasing, bathtub-shaped, or increasing then bathtub-shaped forms (see Glaser [24] or Theorem 2.8 in the study by Lai and Xie [25]). Figure 2 draws the failure rate function for some values of the parameters and shows possible forms of it graphically.

Proposition 1. The $M R L$ function of $X \sim E L(k, m ; \delta, \theta)$ is

$$
m(x)=\frac{1}{\theta} \frac{\Gamma(k) \Gamma(\theta x, m+1)+\delta \Gamma(\theta x, k+1)}{\Gamma(k) \Gamma(\theta x, m)+\delta \Gamma(\theta x, k)}-x .
$$

Proof. The MRL can be expressed by

$$
m(x)=\frac{\int_{x}^{\infty} R(t) \mathrm{d} t}{R(x)} .
$$

The numerator of (8) can be simplified as

$\int_{x}^{\infty} R(t) \mathrm{d} t=\frac{1}{\Gamma(m)+\delta} \int_{x}^{\infty} \Gamma(\theta t, m)+\frac{\delta}{\Gamma(k)} \Gamma(\theta t, k) \mathrm{d} t$.

However,

$$
\begin{aligned}
\int_{x}^{\infty} \Gamma(\theta t, k) \mathrm{d} t & =\int_{x}^{\infty} \int_{\theta t}^{\infty} y^{k-1} e^{-y} \mathrm{~d} y \mathrm{~d} t \\
& =\int_{\theta x}^{\infty} \int_{x}^{\frac{y}{\theta}} y^{k-1} e^{-y} \mathrm{~d} t \mathrm{~d} y \\
& =\int_{\theta x}^{\infty} y^{(k+1)-1} e^{-y} \mathrm{~d} y-x \int_{\theta x}^{\infty} y^{k-1} e^{-y} \mathrm{~d} y \\
& =\frac{1}{\theta} \Gamma(\theta x, k+1)-x \Gamma(\theta x, k) .
\end{aligned}
$$

Similarly,

$$
\int_{x}^{\infty} \Gamma(\theta t, m) \mathrm{d} t=\frac{1}{\theta} \Gamma(\theta x, m+1)-x \Gamma(\theta x, m) .
$$

Then, by (8)-(11), the result follows.

The $\alpha$-QRL is defined to be

$$
q_{\alpha}(x)=\inf \{y: R(y+x)=\bar{\alpha} R(x)\}, \quad x \geq 0,
$$

or more abbreviate $q_{\alpha}(x)=R^{-1}(\bar{\alpha} R(x))-x$. When $X \sim \operatorname{EL}(k, m ; \delta, \theta)$, the $R^{-1}$ has not closed form and should be calculated numerically. For $\alpha=0.5$, it is referred to median residual life which is an alternative for MRL in the reliability and survival analysis literature.

In Figure 3, left side, we use (7) to draw the MRL. Also, in the right side of Figure 3, the median residual life has been plotted for some parameters.

Since the failure rate function may be increasing, bathtub-shaped, or increasing, then bathtub-shaped, the MRL function, and the $\alpha$-QRL function may be decreasing, upside down bathtub-shaped, or decreasing then upside down bathtub-shaped, respectively (refer to Lai and Xie [25]). Figure 3 confirms this result graphically.

The point which maximizes the MRL/median residual life function is known as the burn-in time and has been attracted interest of many authors (see Mi [26]). 

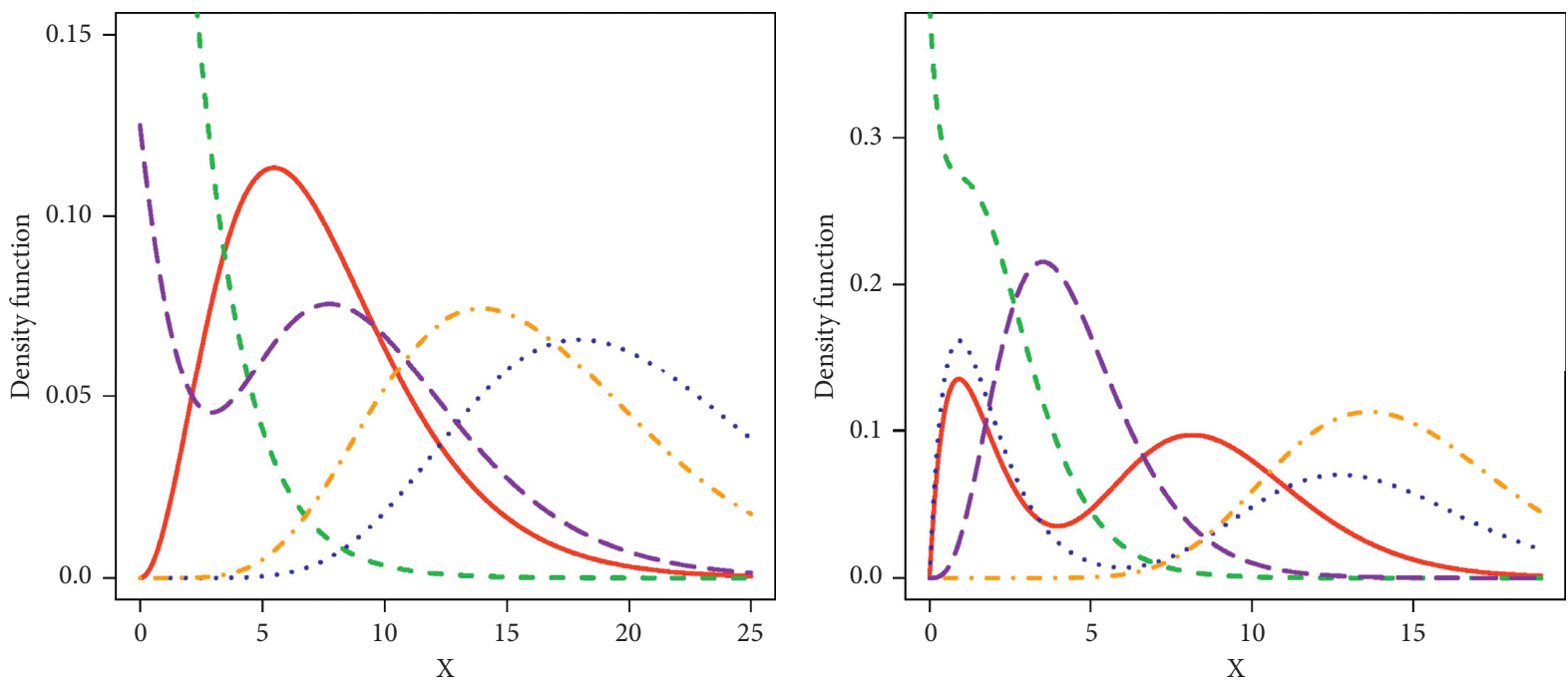

$$
\begin{aligned}
& -\mathrm{k}=3, \mathrm{~m}=4, \delta=2, \theta=0.5 \\
& --\mathrm{k}=1, \mathrm{~m}=1, \delta=1, \theta=0.5 \\
& \cdots \cdots \mathrm{k}=4, \mathrm{~m}=10, \delta=0.7, \theta=0.5 \\
& \cdots-\mathrm{k}=3, \mathrm{~m}=8, \delta=1.5, \theta=0.5 \\
& --\mathrm{k}=5, \mathrm{~m}=1, \delta=3, \theta=0.5
\end{aligned}
$$

(a)

$$
\begin{aligned}
& -\mathrm{k}=10, \mathrm{~m}=2, \delta=2, \theta=1.1 \\
& --\mathrm{k}=1, \mathrm{~m}=3, \delta=1.1, \theta=1.1 \\
& \cdots \cdots \mathrm{k}=15, \mathrm{~m}=2, \delta=1.5, \theta=1.1 \\
& \cdots \mathrm{k}=2, \mathrm{~m}=16, \delta=3, \theta=1.1 \\
& --\mathrm{k}=4, \mathrm{~m}=5, \delta=4, \theta=1.1
\end{aligned}
$$

(b)

FIgURE 1: The PDF of $\operatorname{EL}(k, m ; \delta, \theta)$ for some values of parameters.
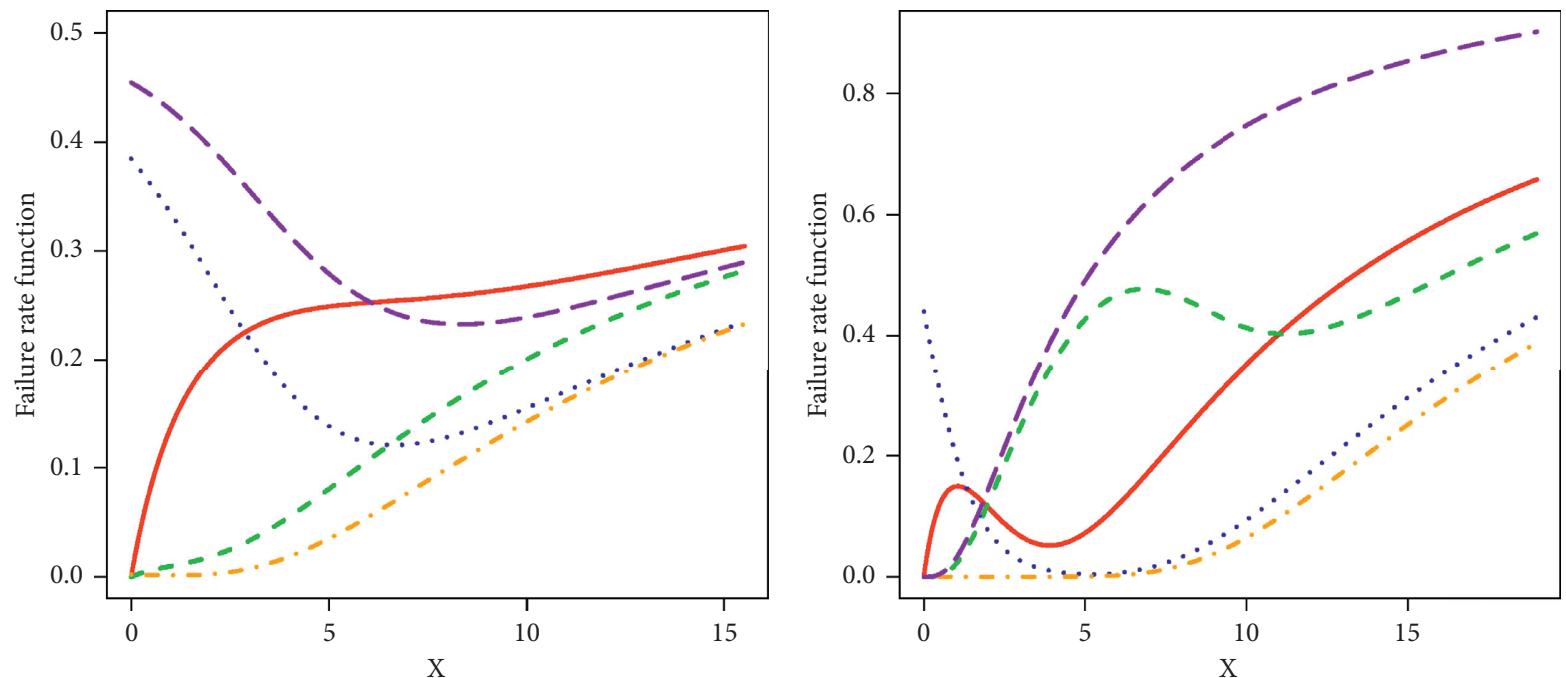

$$
\begin{aligned}
&-\mathrm{k}=5, \mathrm{~m}=2, \delta=0.2, \theta=0.5 \\
&--\mathrm{k}=2, \mathrm{~m}=5, \delta=1.5, \theta=0.5 \\
& \cdots \cdots \mathrm{k}=6, \mathrm{~m}=1, \delta=0.3, \theta=0.5 \\
&-\cdot \mathrm{k}=1, \mathrm{~m}=6, \delta=0.5, \theta=0.5 \\
&--\mathrm{k}=5, \mathrm{~m}=1, \delta=0.1, \theta=0.5
\end{aligned}
$$

(a)

$$
\begin{aligned}
& -\mathrm{k}=10, \mathrm{~m}=2, \delta=2, \theta=1.1 \\
& --\mathrm{k}=12, \mathrm{~m}=5, \delta=1.1, \theta=1.1 \\
& \cdots \cdots \mathrm{k}=15, \mathrm{~m}=1, \delta=1.5, \theta=1.1 \\
& --\mathrm{k}=2, \mathrm{~m}=16, \delta=3, \theta=1.1 \\
& --\mathrm{k}=4, \mathrm{~m}=5, \delta=4, \theta=1.1
\end{aligned}
$$

(b)

FIgURE 2: The failure rate function of $\operatorname{EL}(k, m ; \delta, \theta)$ for some values of parameters. 


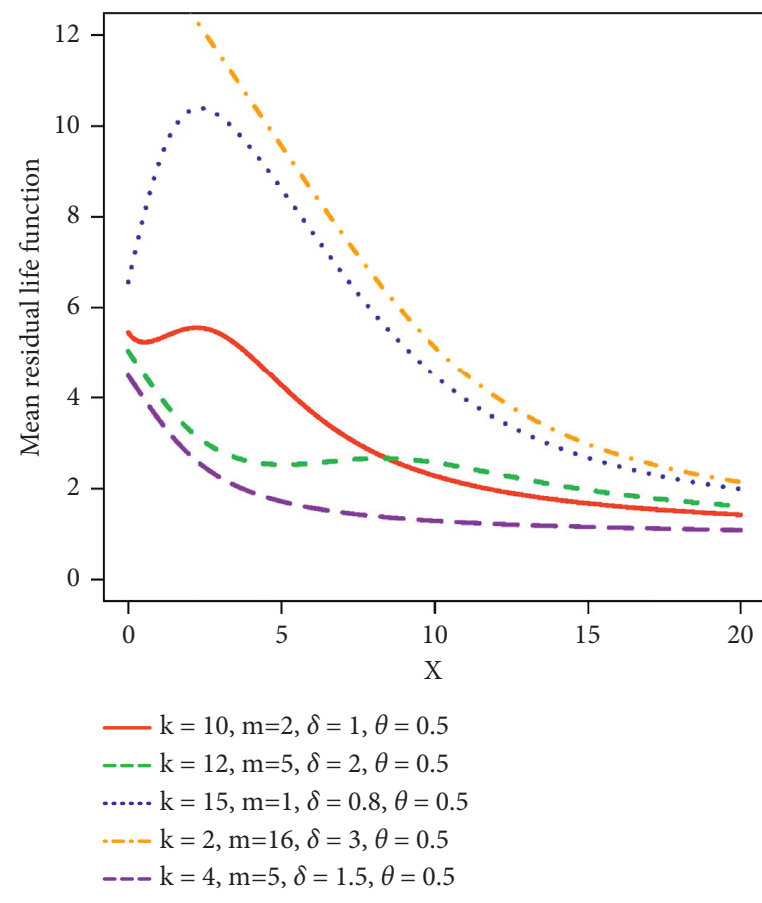

(a)

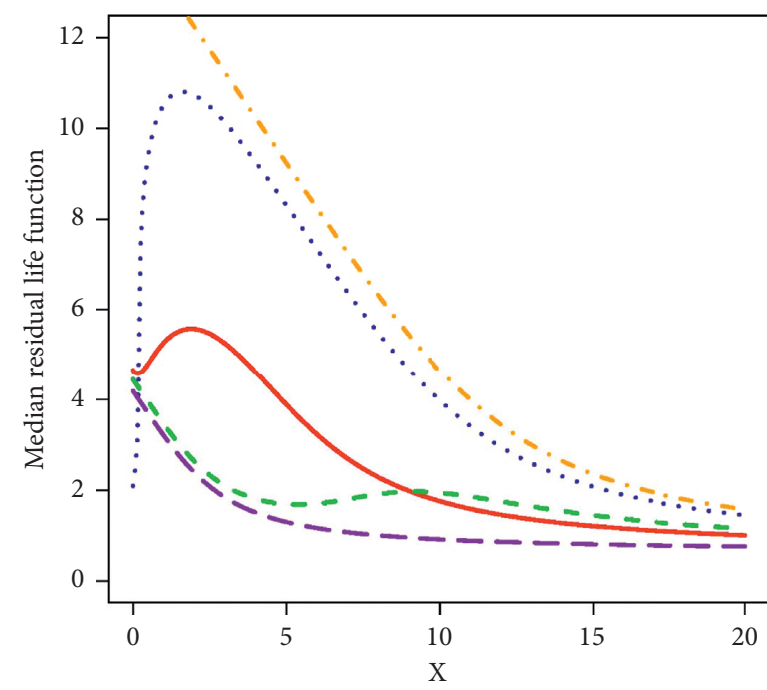

$$
\begin{aligned}
& -\mathrm{k}=10, \mathrm{~m}=2, \delta=1, \theta=1.1 \\
& ---\mathrm{k}=12, \mathrm{~m}=5, \delta=2, \theta=1.1 \\
& \cdots \cdots \mathrm{k}=15, \mathrm{~m}=1, \delta=0.8, \theta=1.1 \\
& -\cdot \mathrm{k}=2, \mathrm{~m}=16, \delta=3, \theta=1.1 \\
& --\mathrm{k}=4, \mathrm{~m}=5, \delta=1.5, \theta=1.1
\end{aligned}
$$

(b)

Figure 3: (a) The MRL of $\operatorname{EL}(k, m ; \delta, \theta)$ for some values of parameters. (b) The median residual life of $\operatorname{EL}(k, m ; \delta, \theta)$ for some parameter values.

\section{Power Extended Lindley}

Now, we introduce the power Lindley extended (PEL) distribution with parameters $k>0, m>0, k \neq m, \delta>0, \theta>0$, and $\gamma>0$, namely, $\operatorname{PEL}(k, m ; \delta, \theta, \gamma)$, with the $\operatorname{PDF}$ as follows:

$$
f(x)=\frac{\theta \gamma x^{\gamma-1}}{\Gamma(m)+\delta}\left(\delta \frac{\left(\theta x^{\gamma}\right)^{k-1}}{\Gamma(k)}+\left(\theta x^{\gamma}\right)^{m-1}\right) e^{-\theta x^{\gamma}}, \quad x \geq 0 .
$$

For $m=2, k=1, \theta=\delta$, and $\gamma=1$, it is the Lindley model. On the other hand, when $\delta=0$ and $\gamma=1$, it reduces to the gamma distribution with PDF as follows:

$$
f(x)=\frac{\theta^{m}}{\Gamma(m)} x^{m-1} e^{-\theta x}, \quad x>0 .
$$

If $Y \sim \operatorname{EL}(k, m ; \delta, \theta)$, then $X=Y^{1 / \gamma} \sim \operatorname{PEL}(k, m ; \delta, \theta, \gamma)$. This statement is useful for simulating random samples of $\operatorname{PEL}(k, m ; \delta, \theta, \gamma)$ and studying its properties.
Figure 4 shows the PDF for some various values of the parameters and indicates that the PDF accommodates decreasing, unimodal, and biomodal forms.

It is straightforward to show that the $\mathrm{CDF}$ of $\operatorname{PEL}(k, m ; \delta, \theta, \gamma)$ is

$$
F(x)=1-\frac{1}{\Gamma(m)+\delta}\left(\frac{\delta}{\Gamma(k)} \Gamma\left(\theta x^{\gamma}, k\right)+\Gamma\left(\theta x^{\gamma}, m\right)\right), \quad x \geq 0,
$$

in which $\Gamma(u, \alpha)=\int_{u}^{\infty} t^{\alpha-1} e^{-t} \mathrm{~d} t$ is the upper incomplete gamma function. The $r^{\text {th }}$ moment of $X \sim \operatorname{PEL}(k, m ; \delta, \theta, \gamma)$ is

$$
E\left(X^{r}\right)=\theta^{-(r / \gamma)} \frac{1}{\Gamma(m)+\delta}\left(\frac{\delta}{\Gamma(k)} \Gamma\left(k+\frac{r}{\gamma}\right)+\Gamma\left(m+\frac{r}{\gamma}\right)\right) .
$$

It is clear that when $k>1$ and $m>1, E\left(X^{r}\right)>\theta^{-(r / \gamma)}$. By the fact that $e^{t X}=\sum_{j=0}^{\infty}(t X)^{j} / j$ ! and applying (16), the moment-generating function can be simplified to

$$
E\left(e^{t X}\right)=\sum_{j=0}^{\infty} \frac{t^{j}}{j !} \theta^{-(j / \gamma)} \frac{1}{\Gamma(m)+\delta}\left(\frac{\delta}{\Gamma(k)} \Gamma\left(k+\frac{j}{\gamma}\right)+\Gamma\left(m+\frac{j}{\gamma}\right)\right) .
$$


3.1. Dynamic Measures. Let $X \sim \operatorname{PEL}(k, m ; \delta, \theta, \gamma)$ with the reliability function $R(x)$, then the failure rate function is

$$
h(x)=\frac{f(x)}{R(x)}=\frac{\delta\left(\theta x^{\gamma}\right)^{k-1}+\Gamma(k)\left(\theta x^{\gamma}\right)^{m-1}}{\delta \Gamma\left(\theta x^{\gamma}, k\right)+\Gamma(k) \Gamma\left(\theta x^{\gamma}, m\right)} \theta \gamma x^{\gamma-1} e^{-\theta x^{\gamma}} .
$$

This failure rate function will accommodate increasing, bathtub, and increasing then bathtub-shaped forms since it is a power model of the baseline model $\operatorname{EL}(k, m ; \delta, \theta)$. The form of the failure rate of the power model depends on the baseline model and the value of $\gamma$. For example, if the failure rate function of the baseline model be increasing and $\gamma>1$, then the failure rate function of the power model will be clearly increasing. If the failure rate function of the baseline model shows bathtub shape and $\gamma>1$, then the failure rate function of the power model will be bathtub-shaped or increasing and so on.

Figure 5 draws the failure rate function for some values of parameters and confirms that it accommodates increasing, bathtub, and increasing then bathtub-shaped forms graphically.
Proposition 2. The MRL function of $X \sim P E L(k, m ; \delta, \theta, \gamma)$ is

$$
m(x)=\theta^{-(1 / \gamma)} \frac{\delta \Gamma\left(\theta x^{\gamma}, k+(1 / \gamma)\right)+\Gamma(k) \Gamma\left(\theta x^{\gamma}, m+(1 / \gamma)\right)}{\delta \Gamma\left(\theta x^{\gamma}, k\right)+\Gamma(k) \Gamma\left(\theta x^{\gamma}, m\right)}-x
$$

Proof. The MRL can be expressed by

$$
m(x)=\frac{\int_{x}^{\infty} R(t) \mathrm{d} t}{R(x)} .
$$

The numerator of (20) can be simplified as

$$
\int_{x}^{\infty} R(t) \mathrm{d} t=\frac{1}{\Gamma(m)+\delta} \int_{x}^{\infty} \frac{\delta}{\Gamma(k)} \Gamma\left(\theta t^{\gamma}, k\right)+\Gamma\left(\theta t^{\gamma}, m\right) \mathrm{d} t
$$

However,

$$
\begin{aligned}
\int_{x}^{\infty} \Gamma\left(\theta t^{\gamma}, k\right) \mathrm{d} t & =\int_{x}^{\infty} \int_{\theta t^{\gamma}}^{\infty} y^{k-1} e^{-y} \mathrm{~d} y \mathrm{~d} t=\int_{\theta x^{\gamma}}^{\infty} \int_{x}^{(y / \theta)^{1 / \gamma}} y^{k-1} e^{-y} \mathrm{~d} t \mathrm{~d} y \\
& =\theta^{-(1 / \gamma)} \int_{\theta x^{\gamma}}^{\infty} y^{(k+(1 / \gamma))-1} e^{-y} \mathrm{~d} y-x \int_{\theta x^{\gamma}}^{\infty} y^{k-1} e^{-y} \mathrm{~d} y=\theta^{-(1 / \gamma)} \Gamma\left(\theta x^{\gamma}, k+\frac{1}{\gamma}\right)-x \Gamma\left(\theta x^{\gamma}, k\right) .
\end{aligned}
$$

Similarly,

$$
\int_{x}^{\infty} \Gamma\left(\theta t^{\gamma}, m\right) \mathrm{d} t=\theta^{-(1 / \gamma)} \Gamma\left(\theta x^{\gamma}, m+\frac{1}{\gamma}\right)-x \Gamma\left(\theta x^{\gamma}, m\right) .
$$

Then, by (20)-(23), the result follows.

The $\alpha$-QRL is defined to be

$$
q_{\alpha}(x)=\inf \{y: R(y+x)=\bar{\alpha} R(x)\}, \quad x \geq 0,
$$

or more abbreviated, $q_{\alpha}(x)=R^{-1}(\bar{\alpha} R(x))-x$. When $X \sim \operatorname{PEL}(k, m ; \delta, \theta, \gamma)$, the $R^{-1}$ has not closed form and should be calculated numerically.

Similarly, since the failure rate function shows increasing, bathtub-shaped, or increasing then bathtub-shaped, the MRL function and the $\alpha$-QRL function may be decreasing, upside down bathtub-shaped, or decreasing then upside down bathtub-shaped, respectively (see Lai and Xie [25]). Figures 6 and 7 show the MRL and the median residual life functions for some parameters and confirm the mentioned forms graphically.

\section{Parameter Estimation}

Let $X_{i}, i=1,2, \ldots, n$ represent an independent and identically distributed (iid) sample from $\operatorname{EL}(k, m ; \delta, \theta)$, and $k$ and $m$ are known. Via the moment method, by $(5),(\delta, \theta)$ can be estimated by solving the following equations in terms of them:

$$
\left\{\begin{array}{l}
\bar{X}=\frac{1}{\theta} \frac{1}{\Gamma(m)+\delta}(\Gamma(m+1)+k \delta) \\
\overline{X^{2}}=\frac{1}{\theta^{2}} \frac{1}{\Gamma(m)+\delta}(\Gamma(m+2)+k(k+1) \delta) .
\end{array}\right.
$$

For $m \geq 1$ and $k \geq 1$, the right side of these equations is greater than $1 / \theta$ and $1 / \theta^{2}$, respectively. So, one lower bound for $\theta$ is $\max \left((1 / \bar{X}),\left(1 / \overline{X^{2}}\right)^{0.5}\right)$.

The log-likelihood function for $(\delta, \theta)$ when $k$ and $m$ are known is

$$
l(\delta, \theta ; \mathbf{X})=n \ln (\theta)-n \ln (\Gamma(m)+\delta)+\sum_{i=1}^{n} \ln \left(\frac{\delta}{\Gamma(k)}\left(\theta X_{i}\right)^{k-1}+\left(\theta X_{i}\right)^{m-1}\right)-\theta \sum_{i=1}^{n} X_{i}
$$



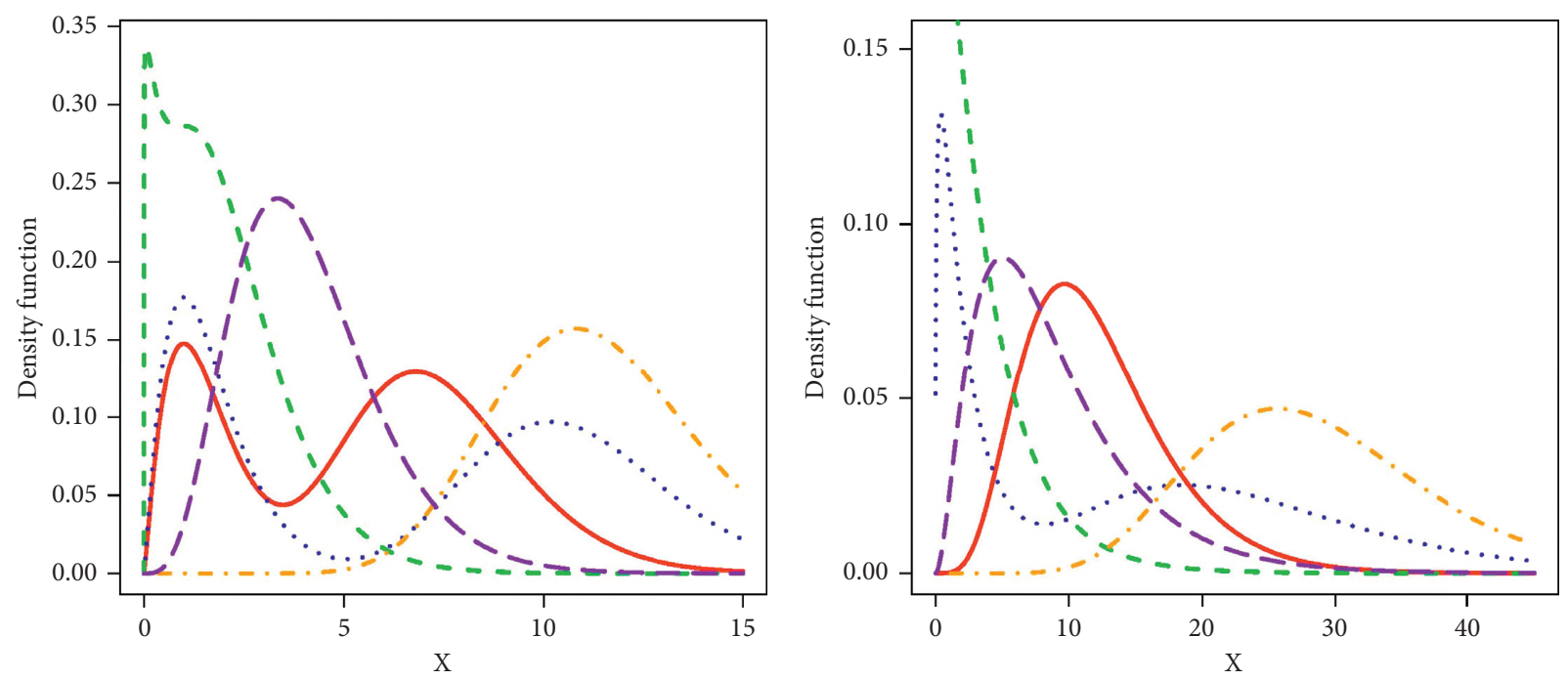

$$
\begin{aligned}
& -\mathrm{k}=10, \mathrm{~m}=2, \delta=2, \theta=1.1, \gamma=1.1 \\
& --\mathrm{k}=1, \mathrm{~m}=3, \delta=1.1, \theta=1.1, \gamma=1.05 \\
& \cdots \cdots \mathrm{k}=15, \mathrm{~m}=2, \delta=1.5, \theta=1.1, \gamma=1.1 \\
& -\cdot \mathrm{k}=2, \mathrm{~m}=16, \delta=3, \theta=1.1, \gamma=1.1 \\
& --\mathrm{k}=4, \mathrm{~m}=5, \delta=4, \theta=1.1, \gamma=1.05
\end{aligned}
$$

(a)

$$
\begin{aligned}
& -\mathrm{k}=3, \mathrm{~m}=8, \delta=2, \theta=1.1, \gamma=0.8 \\
& --\mathrm{k}=1, \mathrm{~m}=3, \delta=1.1, \theta=1.1, \gamma=0.7 \\
& \cdots \cdots \mathrm{k}=10, \mathrm{~m}=2, \delta=1.5, \theta=1.1, \gamma=0.7 \\
& \cdots-\mathrm{k}=2, \mathrm{~m}=16, \delta=3, \theta=1.1, \gamma=0.8 \\
& --\mathrm{k}=4, \mathrm{~m}=5, \delta=4, \theta=1.1, \gamma=0.7
\end{aligned}
$$

(b)

Figure 4: The PDF of $\operatorname{PEL}(k, m ; \delta, \theta, \gamma)$ for some values of the parameters.

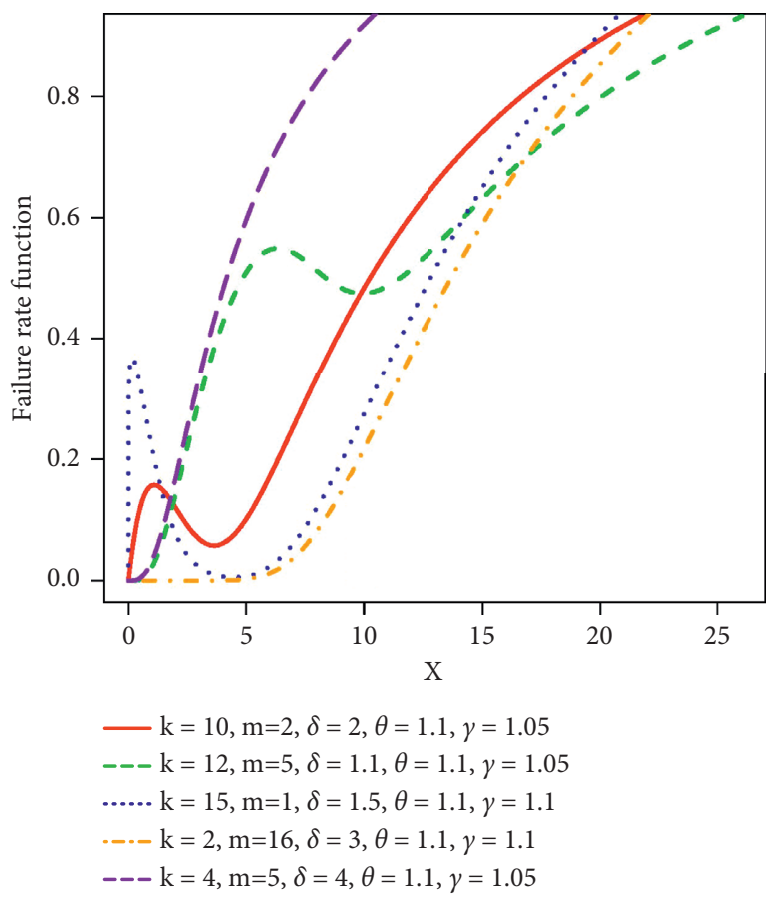

(a)

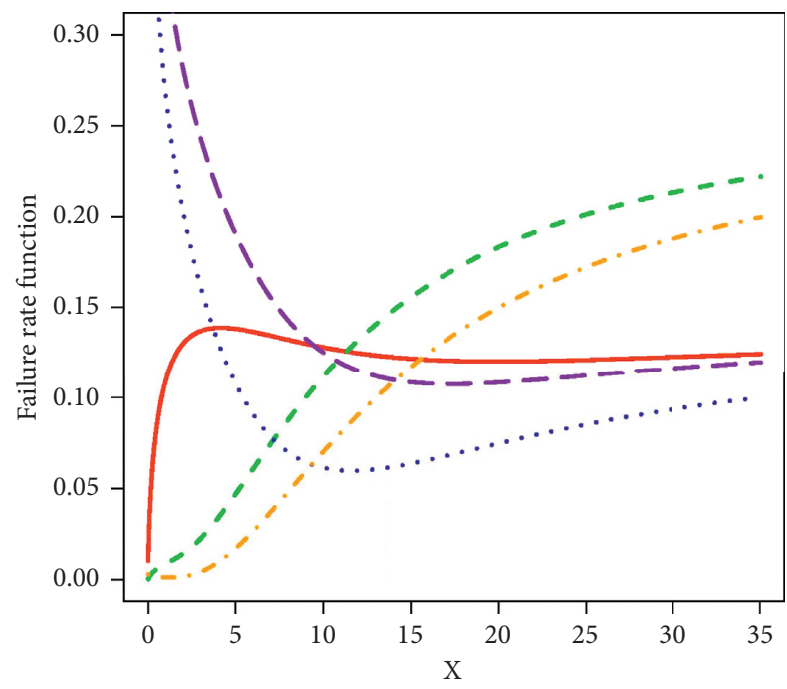

$\begin{aligned} &-\mathrm{k}=5, \mathrm{~m}=2, \delta=0.2, \theta=0.5, \gamma=0.8 \\ &--\mathrm{k}=2, \mathrm{~m}=5, \delta=1.5, \theta=0.5, \gamma=0.9 \\ & \cdots \cdots \mathrm{k}=6, \mathrm{~m}=1, \delta=0.3, \theta=0.5, \gamma=0.8 \\ &-\cdot \mathrm{k}=1, \mathrm{~m}=6, \delta=0.5, \theta=0.5, \gamma=0.9 \\ &--\mathrm{k}=5, \mathrm{~m}=1, \delta=0.1, \theta=0.5, \gamma=0.8\end{aligned}$

(b)

Figure 5: The failure rate function of $\operatorname{PEL}(k, m ; \delta, \theta, \gamma)$ for some values of the parameters. 

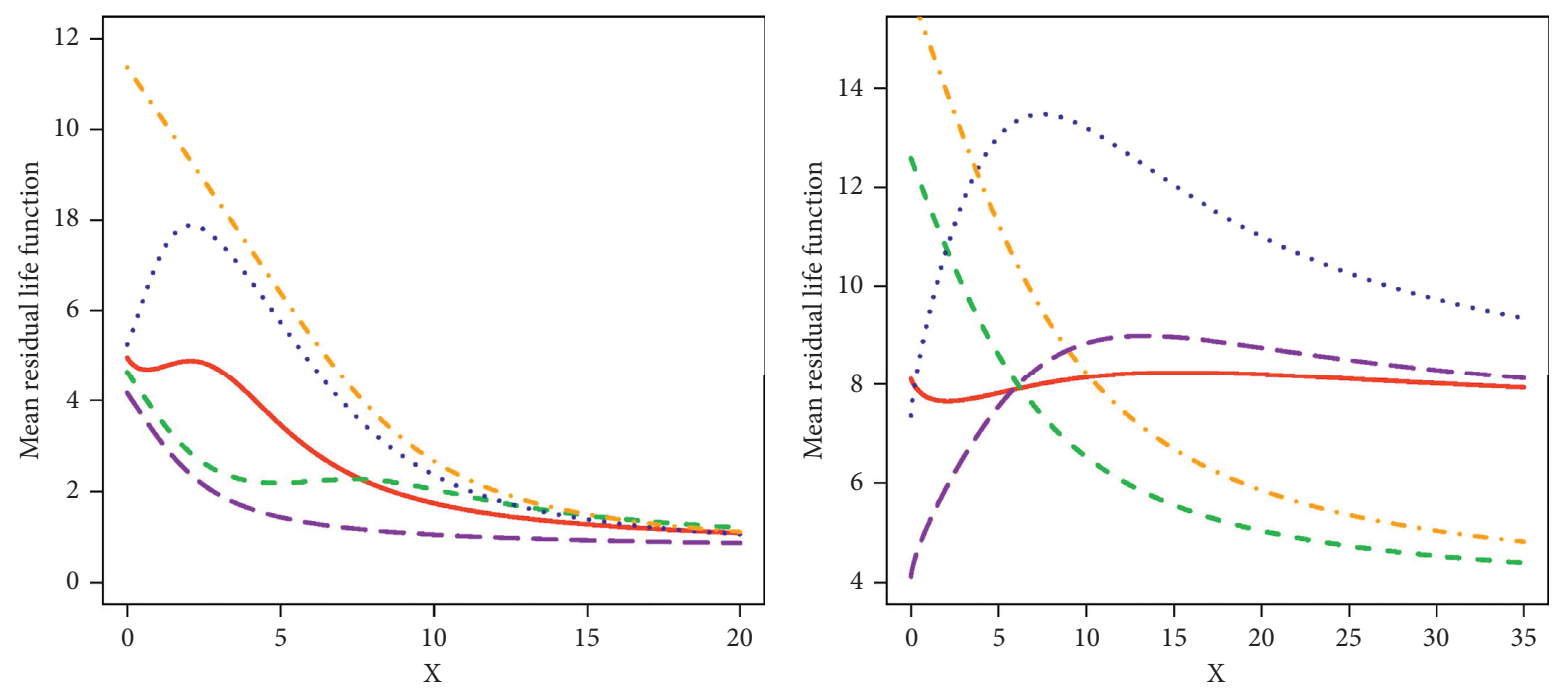

$-\mathrm{k}=10, \mathrm{~m}=2, \delta=1, \theta=1.1, \gamma=1.05$
$---\mathrm{k}=12, \mathrm{~m}=5, \delta=2, \theta=1.1, \gamma=1.05$
$\cdots \cdots \mathrm{k}=15, \mathrm{~m}=1, \delta=0.8, \theta=1.1, \gamma=1.1$
$\cdots-\mathrm{k}=2, \mathrm{~m}=16, \delta=3, \theta=1.1, \gamma=1.1$
$--\mathrm{k}=4, \mathrm{~m}=5, \delta=1.5, \theta=1.1, \gamma=1.05$

(a)

$$
\begin{aligned}
&-\mathrm{k}=5, \mathrm{~m}=2, \delta=0.2, \theta=0.5, \gamma=0.8 \\
&--\mathrm{k}=2, \mathrm{~m}=5, \delta=1.5, \theta=0.5, \gamma=0.9 \\
& \cdots . \mathrm{k}=6, \mathrm{~m}=1, \delta=0.3, \theta=0.5, \gamma=0.8 \\
& \cdots-\mathrm{k}=1, \mathrm{~m}=6, \delta=0.5, \theta=0.5, \gamma=0.9 \\
&--\mathrm{k}=5, \mathrm{~m}=1, \delta=0.1, \theta=0.5, \gamma=0.8
\end{aligned}
$$

(b)

FIgURE 6: Some forms of the MRL of $\operatorname{PEL}(k, m ; \delta, \theta, \gamma)$.
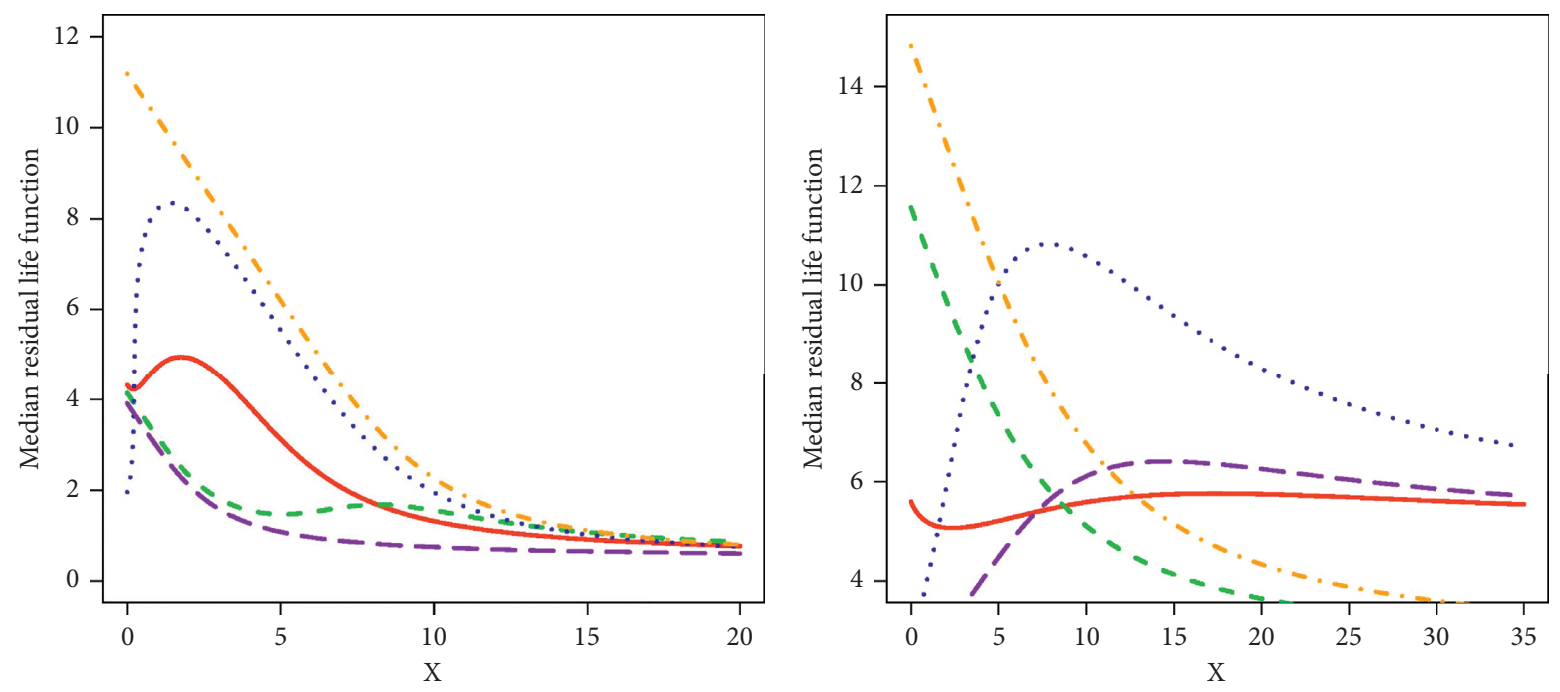

$-\mathrm{k}=10, \mathrm{~m}=2, \delta=1, \theta=1.1, \gamma=1.05$
$---\mathrm{k}=12, \mathrm{~m}=5, \delta=2, \theta=1.1, \gamma=1.05$
$\cdots \cdots \mathrm{k}=15, \mathrm{~m}=1, \delta=0.8, \theta=1.1, \gamma=1.1$
$\cdots-\mathrm{k}=2, \mathrm{~m}=16, \delta=3, \theta=1.1, \gamma=1.1$
$--\mathrm{k}=4, \mathrm{~m}=5, \delta=1.5, \theta=1.1, \gamma=1.05$

(a)

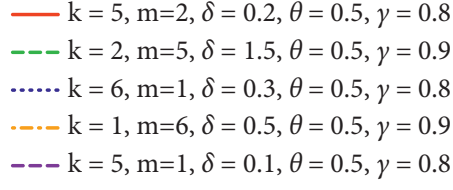

(b)

FIgURE 7: Some forms of the median residual life function of $\operatorname{PEL}(k, m ; \delta, \theta, \gamma)$. 
The log-likelihood equations are

$$
\begin{aligned}
& \frac{\partial l(\delta, \theta ; \mathbf{X})}{\partial \delta}=-\frac{n}{\Gamma(m)+\delta}+\sum_{i=1}^{n} \frac{\left(\theta X_{i}\right)^{k-1}}{\Gamma(k)\left(\theta X_{i}\right)^{m-1}+\delta\left(\theta X_{i}\right)^{k-1}}=0, \\
& \frac{\partial l(\delta, \theta ; \mathbf{X})}{\partial \theta}=\frac{n}{\theta}+\sum_{i=1}^{n} \frac{\Gamma(k)(m-1) X_{i}\left(\theta X_{i}\right)^{m-2}+\delta(k-1) X_{i}\left(\theta X_{i}\right)^{k-2}}{\Gamma(k)\left(\theta X_{i}\right)^{m-1}+\delta\left(\theta X_{i}\right)^{k-1}}-\sum_{i=1}^{n} X_{i}=0 .
\end{aligned}
$$

The MLE can be computed by directly maximizing (26) or solving the log-likelihood equations (27) and (28) simultaneously.

The Fisher information matrix in one sample of size $n$ about $(\delta, \theta)$ is

$$
K=\left[\begin{array}{cc}
E\left(-\frac{\partial^{2} l}{\partial \delta^{2}}\right) & E\left(-\frac{\partial^{2} l}{\partial \delta \partial \theta}\right) \\
E\left(-\frac{\partial^{2} l}{\partial \theta \partial \delta}\right) & E\left(-\frac{\partial^{2} l}{\partial \theta^{2}}\right)
\end{array}\right],
$$

where $l=\ln f(X)$. Let $K^{-1}$ be the inverse of the information matrix, then $\operatorname{Var}(\widehat{\delta}, \widehat{\theta}) \approx n^{-1} K^{-1}$. Moreover, if $X_{i}$, $i=1,2, \ldots, n$ represents an iid sample from $\operatorname{EL}\left(k, m ; \delta_{0}, \theta_{0}\right)$, then

$$
\sqrt{n}\left(\widehat{\delta}-\delta_{0}, \widehat{\theta}-\theta_{0}\right)^{T} \sim N\left(0, K^{-1}\right),
$$

asymptotically, which can be applied for constructing confidence intervals for the parameters or in hypothesis testing.

When $k$ and/or $m$ are unknown which is usually the case, we can estimate $(\delta, \theta)$ for a suitable set of values of $k$ and $m$ and compare the estimated distribution to the empirical distribution by applying statistics like Kolmogorov-Smirnov (K-S) statistic.

4.1. Parameter Estimation of the Power Extended Lindley. Let $X_{i}, \quad i=1,2, \ldots, n$ represent an iid sample from $\operatorname{PEL}(k, m ; \delta, \theta, \gamma)$, and $k$ and $m$ are known. Via the moment method, by (16), the triple $(\delta, \theta, \gamma)$ can be estimated by finding their solution in the following system of equations:

$$
\left\{\begin{array}{l}
\bar{X}=\theta^{-(1 / \gamma)} \frac{1}{\Gamma(m)+\delta}\left(\frac{\delta}{\Gamma(k)} \Gamma\left(k+\frac{1}{\gamma}\right)+\Gamma\left(m+\frac{1}{\gamma}\right)\right), \\
\overline{X^{2}}=\theta^{-(2 / \gamma)} \frac{1}{\Gamma(m)+\delta}\left(\frac{\delta}{\Gamma(k)} \Gamma\left(k+\frac{2}{\gamma}\right)+\Gamma\left(m+\frac{2}{\gamma}\right)\right), \\
\overline{X^{3}}=\theta^{-(3 / \gamma)} \frac{1}{\Gamma(m)+\delta}\left(\frac{\delta}{\Gamma(k)} \Gamma\left(k+\frac{3}{\gamma}\right)+\Gamma\left(m+\frac{3}{\gamma}\right)\right) .
\end{array}\right.
$$

The log-likelihood function for $(\delta, \theta, \gamma)$ when $k$ and $m$ are known is

$$
\begin{aligned}
l(\delta, \theta, \gamma ; \mathbf{X})= & n \ln (\gamma)+(\gamma-1) \sum_{i=1}^{n} \ln \left(X_{i}\right)+n \ln (\theta)-n \ln (\Gamma(m)+\delta) \\
& +\sum_{i=1}^{n} \ln \left(\frac{\delta}{\Gamma(k)}\left(\theta X_{i}^{\gamma}\right)^{k-1}+\left(\theta X_{i}^{\gamma}\right)^{m-1}\right)-\theta \sum_{i=1}^{n} X_{i}^{\gamma} .
\end{aligned}
$$

The log-likelihood equations are

$$
\begin{aligned}
& \frac{\partial l(\delta, \theta, \gamma ; \mathbf{X})}{\partial \delta}=-\frac{n}{\Gamma(m)+\delta}+\sum_{i=1}^{n} \frac{\left(\theta X_{i}^{\gamma}\right)^{k-1}}{\Gamma(k)\left(\theta X_{i}^{\gamma}\right)^{m-1}+\delta\left(\theta X_{i}^{\gamma}\right)^{k-1}}=0, \\
& \frac{\partial l(\delta, \theta, \gamma ; \mathbf{X})}{\partial \theta}=\frac{n}{\theta}+\sum_{i=1}^{n} \frac{\Gamma(k)(m-1) X_{i}^{\gamma}\left(\theta X_{i}^{\gamma}\right)^{m-2}+\delta(k-1) X_{i}^{\gamma}\left(\theta X_{i}^{\gamma}\right)^{k-2}}{\Gamma(k)\left(\theta X_{i}^{\gamma}\right)^{m-1}+\delta\left(\theta X_{i}^{\gamma}\right)^{k-1}}-\sum_{i=1}^{n} X_{i}^{\gamma}=0,
\end{aligned}
$$




$$
\frac{\partial l(\delta, \theta, \gamma ; \mathbf{X})}{\partial \gamma}=\frac{n}{\gamma}+\sum_{i=1}^{n} \ln X_{i}+\sum_{i=1}^{n} \ln X_{i} \frac{\delta(k-1)\left(\theta X_{i}^{\gamma}\right)^{k-1}+\Gamma(k)(m-1)\left(\theta X_{i}^{\gamma}\right)^{m-1}}{\delta\left(\theta X_{i}^{\gamma}\right)^{k-1}+\Gamma(k)\left(\theta X_{i}^{\gamma}\right)^{m-1}}-\theta \sum_{i=1}^{n} X_{i}^{\gamma} \ln X_{i}=0
$$

The MLE can be computed by maximizing (32) directly or solving the log-likelihood equations (33)-(35) simultaneously.

The Fisher information matrix in one sample of size $n$ about $(\delta, \theta, \gamma)$ is

$$
K=\left[\begin{array}{ccc}
E\left(-\frac{\partial^{2} l}{\partial \delta^{2}}\right) & E\left(-\frac{\partial^{2} l}{\partial \delta \partial \theta}\right) & E\left(-\frac{\partial^{2} l}{\partial \delta \partial \gamma}\right) \\
E\left(-\frac{\partial^{2} l}{\partial \theta \partial \delta}\right) & E\left(-\frac{\partial^{2} l}{\partial \theta^{2}}\right) & E\left(-\frac{\partial^{2} l}{\partial \theta \partial \gamma}\right) \\
E\left(-\frac{\partial^{2} l}{\partial \gamma \partial \delta}\right) & E\left(-\frac{\partial^{2} l}{\partial \gamma \partial \theta}\right) & E\left(-\frac{\partial^{2} l}{\partial \gamma^{2}}\right)
\end{array}\right],
$$

where $l=\ln f(X)$. Assume that $K^{-1}$ be the inverse of the information matrix, then $\operatorname{Var}(\widehat{\delta}, \widehat{\theta}, \widehat{\gamma}) \approx n^{-1} K^{-1}$. Furthermore, let $X_{i}, i=1,2, \ldots, n$ represent an iid sample from $\operatorname{PEL}\left(k, m ; \delta_{0}, \theta_{0}, \gamma_{0}\right)$, then

$$
\sqrt{n}\left(\widehat{\delta}-\delta_{0}, \widehat{\theta}-\theta_{0}, \widehat{\gamma}-\gamma_{0}\right)^{T} \sim N\left(0, K^{-1}\right),
$$

asymptotically, which can be applied for constructing confidence intervals for the parameters or in hypothesis testing.

Similarly, when $k$ and/or $m$ are unknown, we can estimate $(\delta, \theta, \gamma)$ for a suitable set of values of $k$ and $m$ and compare the estimated distribution to the empirical distribution by K-S statistic or other similar statistics.

4.2. Right-Censored Data. Assume that we have one iid random sample $X_{i}, i=1,2, \ldots, n$ from the EL (PEL) which is exposed to right censorship. Then, $X_{i}$ is censored from right by a censoring random variable $C_{i}$, if $C_{i} \leq X_{i}$, so the only information about event time is that it is greater than censoring time $C_{i}$. Thus, the observations consist of $T_{i}=$ $\min \left(X_{i}, C_{i}\right)$ and $d_{i}$, where $d_{i}=1$, when the event is not censored, $X_{i} \leq C_{i}$, and $d_{i}=0$, when the event is censored, $X_{i}>C_{i}$. With the censored data in hand, the log-likelihood function is denoted by $l_{c}(\delta, \theta ; t, d)\left(l_{c}(\delta, \theta, \gamma ; t, d)\right)$ and equals to

$$
\sum_{i=1}^{n} d_{i} \ln f\left(t_{i}\right)+\sum_{i=1}^{n}\left(1-d_{i}\right) \ln R\left(t_{i}\right)
$$

where $f$ and $R$ show the density and the reliability functions of the EL (PEL), respectively. We can maximize this function in terms of $(\delta, \theta)(\delta, \theta, \gamma)$ to find the MLE and estimate its variance by reverse of the Fisher information matrix.

\section{Simulation Study}

The proposed distribution is a mixture of two gamma distribution, so we can follow the below steps to generate random samples of size $n$ of it.

(1) Simulate one random instance from binomial distribution with parameters $n$ and $\delta / \Gamma(m)+\delta$. Let $n_{1}$ be the generated instance and $n_{2}=n-n_{1}$.

(2) Simulate one random sample of size $n_{1}$ from $G(k, \theta)$ and one random sample of size $n_{2}$ from $G(m, \theta)$. Then, merge these two sample to provide one random sample from $\operatorname{EL}(k, m, \delta, \theta)$.

(3) To simulate one sample of size $n$ from $\operatorname{PEL}(k, m ; \delta, \theta, \gamma)$, we simulate one sample from $\operatorname{EL}(k, m, \delta, \theta)$ as described in the previous steps. Let $Y_{1}, Y_{2}, \ldots, Y_{n}$ show the simulated sample from $\operatorname{EL}(k, m, \delta, \theta)$. Then, we raise this sample to power $1 / \gamma$, i.e., $Y_{1}^{1 / \gamma}, Y_{2}^{1 / \gamma}, \ldots, Y_{n}^{1 / \gamma}$.

(4) In the case that the sample must be censored from right, we assume that $C_{i}$ follows the uniform distribution on the interval $(0, M)$. Given the censorship rate, $p$ and $M$ can be computed through solving the following equation:

$$
\int_{0}^{M} R(t) \mathrm{d} t=M p
$$

where $R$ is the reliability function of the extended Lindley.

The result of this investigation for the EL model is abstracted in Table 1. For every cell of this table, $r=500$ replicates have been simulated. For each replication, the MLE of $(\delta, \theta),(\widehat{\delta}, \widehat{\theta})$, has been computed. Then, three pairs $\left(B_{\delta}, B_{\theta}\right),\left(A B_{\delta}, A B_{\theta}\right)$, and $\left(\mathrm{MSE}_{\delta}, \mathrm{MSE}_{\theta}\right)$ in which

$$
\begin{aligned}
B_{\delta} & =\frac{1}{r} \sum_{i=1}^{r}\left(\widehat{\delta}_{i}-\delta\right), \\
A B_{\delta} & =\frac{1}{r} \sum_{i=1}^{r}\left|\widehat{\delta}_{i}-\delta\right|, \\
\mathrm{MSE}_{\delta} & =\frac{1}{r} \sum_{i=1}^{r}\left(\widehat{\delta}_{i}-\delta\right)^{2},
\end{aligned}
$$

and $B_{\theta}, A B_{\theta}$, and $\mathrm{MSE}_{\theta}$ are defined similarly have been calculated. Results of the simulations in Table 1 indicate that MLE of $(\delta, \theta)$ is efficient and consistent. Moreover, the following observations are notable: 
TABLE 1: Simulation results for MLE of $\theta$ from $\operatorname{EL}(k, m, \delta, \theta)$. Every cell consists of pairs $\left(B_{\delta}, B_{\theta}\right),\left(A B_{\delta}, A B_{\theta}\right)$, and $\left(\mathrm{MSE}_{\delta}\right.$, MSE $\left.\mathrm{E}_{\theta}\right)$ from top to bottom.

\begin{tabular}{|c|c|c|c|c|c|}
\hline \multirow{2}{*}{$n$} & \multirow{2}{*}{$(k, m)$} & \multicolumn{2}{|c|}{$p=0$} & \multicolumn{2}{|c|}{$p=0.20$} \\
\hline & & $\delta=2, \theta=0.01$ & $\delta=1, \theta=2$ & $\delta=2, \theta=0.01$ & $\delta=1, \theta=2$ \\
\hline \multirow{6}{*}{100} & & $(0.564546,0.000322)$ & $(0.125512,0.002711)$ & $(0.201321,-0.000122)$ & $(-0.630433,-0.669626)$ \\
\hline & $(3,1)$ & $(0.862838,0.000784)$ & $(0.406587,0.207322)$ & $(0.824443,0.001039)$ & $(0.795519,0.687137)$ \\
\hline & & $(1.237973,0.000001)$ & $(0.311982,0.076444)$ & $(1.428182,0.000002)$ & $(0.773555,0.606325)$ \\
\hline & & $(0.668760,0.000149)$ & $(0.251394,-0.002273)$ & $(0.650087,-0.000270)$ & $(0.371670,-.027948)$ \\
\hline & $(4,2)$ & $(1.145145,0.000577)$ & $(0.666065,0.224162)$ & $(1.559371,0.001144)$ & $(0.886215,0.278360)$ \\
\hline & & $(1.850450,0.0000006)$ & $(0.843101,0.081450)$ & $(7.354127,0.000002)$ & $(2.729726,0.116106)$ \\
\hline \multirow{6}{*}{150} & & $(0.365396,0.000155)$ & $(0.085817,0.008477)$ & $(0.128090,-0.000109)$ & $(-0.528556,-0.556155)$ \\
\hline & $(3,1)$ & $(0.665277,0.000581)$ & $(0.327193,0.171947)$ & $(0.612812,0.000792)$ & $(0.655863,0.594525)$ \\
\hline & & $(0.814574,0.0000005)$ & $(0.184370,0.051351)$ & $(0.740215,0.000001)$ & $(0.572858,0.520272)$ \\
\hline & & $(0.592613,0.000150)$ & $(0.147898,-0.017771)$ & $(0.276481,-0.000337)$ & $(0.107956,-0.043725)$ \\
\hline & $(4,2)$ & $(1.003509,0.000489)$ & $(0.551938,0.187519)$ & $(1.147521,0.000996)$ & $(0.609155,0.227483)$ \\
\hline & & $(1.519897,0.0000004)$ & $(0.543016,0.057536)$ & $(3.144793,0.000002)$ & $(0.695320,0.082204)$ \\
\hline
\end{tabular}

(i) All measures have smaller values for larger sample size. The MLE has a small positive bias for uncensored data which moves toward negative values for censored samples.

(ii) The measures show larger values for larger $\theta s(\delta s)$.

(iii) For censored samples, $A B_{\theta}$ and $\mathrm{MSE}_{\theta}$ are larger. Table 2 contains the results of simulations for the PEL distribution. $k$ and $m$ have been considered to be 4 and 1, respectively. For every cell of this table, $r=500$ replicates have been simulated, and for each replication, the MLE of $(\delta, \theta, \gamma)$ and $(\widehat{\delta}, \widehat{\theta}, \widehat{\gamma})$ has been calculated. Then, the triples $\left(B_{\delta}, B_{\theta}, B_{\gamma}\right)$, $\left(A B_{\delta}, A B_{\theta}, A B_{\gamma}\right)$, and $\left(\mathrm{MSE}_{\delta}, \mathrm{MSE}_{\theta}, \mathrm{MSE}_{\gamma}\right)$ have been calculated. The simulation results indicate that the MLE of $(\delta, \theta, \gamma)$ is efficient and consistent. Moreover, the following observations are notable.

(iv) The MSE and absolute error have smaller values for larger sample size.

(v) For censored cases, the MSE and absolute error show greater values.

(vi)• The measures about $\delta$ show larger values for larger $\delta$. Similar result holds for $\theta$ and $\gamma$.

\section{Applications}

In this section, four data sets, (see Tables 3-6) have been considered. For every data set, the $\operatorname{EL}(k, m ; \delta, \theta)$ and $\operatorname{PEL}(k, m ; \delta, \theta, \gamma)$ have been fitted, for all pairs $(k, m)$ where $k=1,2, \ldots, 10$ and $m=1,2, \ldots, 10$. Then, based on the K-S statistic and AIC, the best values of $k$ and $m$ are selected. In a comparative analysis, the quasi Lindley (QL) and the power quasi Lindley (PQL) distributions have been fitted to these data sets too. The K-S, the Cramér-von Mises (CVM), and the Anderson-Darling (AD) statistics along with their corresponding $p$ values have been computed. Also, the Akaike information criterion (AIC) has been reported.
Table 3 shows the number of cycles to failure for 25 specimens of yarn, considered in Shanker [28]. The results of fit are gathered in Table 7. Clearly, both EL and PEL describe data very well and in a very close competition. Among the compared models, the PEL gives the best fit in terms of K-S, CVM, and AD statistics. However, the AIC of $\mathrm{QL}$ is smaller than others. Figure 8(a) shows the empirical CDF and fitted CDF of EL and PEL and graphically confirms a good fit. Figure 9(a), shows the PDF of the fitted EL and PEL distributions. Also, left side of Figure 10 shows the scaled total time on test (TTT) plot of the estimated EL and PEL distributions and reveals an increasing failure rate model.

The second data set (see Table 4) consists of 100 waiting times of customers of a bank reported by Shanker [28]. Similarly, Table 7 shows the results of fit for this example. The PEL gives the lowest value for the K-S, CVM, and AD statistics. So, PEL outperforms other models from this point of view. The empirical and fitted CDF for EL and PEL has been plotted in Figure 8(b) and confirms a good fit. Also, the estimated PDF and the scaled TTT plot have been drawn in Figures 9 and 10, respectively.

Table 5 shows the cycles to failure for 60 electrical appliances analyzed by Lawless [27]. It can be seen from Table 7 that the PEL gives a better fit than other rivals due to the lower AIC and K-S, CVM, and AD statistics. Figure 11(a) depicts the fit graphically. The estimated PDF and the scaled TTT plot have been plotted in Figures 12 and 13, respectively.

Table 6 shows survival times of a group of patients suffering from head and neck cancer treated by a combination of radiotherapy and chemotherapy, reported by Efron [29]. Again the PEL distribution gives the best fit among compared models. Figure 11(b) shows the empirical and fitted CDF and indicates a good fit for both models apparently. Moreover, Figures 12 and 13 show the estimated PDF and the scaled TTT plots of the EL and PEL models. 
TABLE 2: Simulation results for MLE of the parameters of PEL $(k, m, \delta, \theta, \gamma)$. Every cell consists of triples $\left(B_{\delta}, B_{\theta}, B_{\gamma}\right),\left(A B_{\delta}, A B_{\theta}, A B_{\gamma}\right)$, and $\left(\mathrm{MSE}_{\delta}, \mathrm{MSE}_{\theta}, \mathrm{MSE}_{\gamma}\right.$ ) from top to bottom.

\begin{tabular}{|c|c|c|c|c|}
\hline$p$ & $n$ & $\gamma$ & $\delta=3, \theta=0.05$ & $\delta=2, \theta=0.01$ \\
\hline \multirow{12}{*}{0} & \multirow{6}{*}{100} & \multirow{3}{*}{1.2} & $(-0.102169,-0.007042,0.122675)$ & $(-0.077950,0.000187,0.085795)$ \\
\hline & & & $(1.385808,0.026254,0.189938)$ & $(0.799550,0.007379,0.169717)$ \\
\hline & & & $(3.532168,0.001054,0.074171)$ & $(1.004847,0.000099,0.045673)$ \\
\hline & & \multirow{4}{*}{0.8} & $(-0.510318,-0.011244,0.014736)$ & $(-0.463949,-0.002733,0.125280)$ \\
\hline & & & $(1.310887,0.025964,0.141285)$ & $(0.902582,0.007098,0.158792)$ \\
\hline & & & $(3.075335,0.000937,0.041458)$ & $(1.187285,0.000078,0.041090)$ \\
\hline & & & $(0.033280,-0.003561,0.072078)$ & $(-0.066741,0.000316,0.055330)$ \\
\hline & \multirow{5}{*}{150} & \multirow[t]{3}{*}{1.2} & $(1.124049,0.021518,0.137609)$ & $(0.658367,0.006076,0.130425)$ \\
\hline & & & $(3.265883,0.000760,0.041036)$ & $(0.721868,0.000070,0.029051)$ \\
\hline & & & $(-0.186257,-0.005086,0.065326)$ & $(-0.569158,-0.003036,0.115042)$ \\
\hline & & \multirow[t]{2}{*}{0.8} & $(1.131052,0.022653,0.109088)$ & $(0.832820,0.006230,0.142214)$ \\
\hline & & & $(2.633615,0.000779,0.027734)$ & $(1.004401,0.000059,0.035583)$ \\
\hline \multirow{12}{*}{0.2} & \multirow{7}{*}{100} & \multirow{4}{*}{1.2} & $(-0.158072,-0.003071,0.125427)$ & $(-0.136315,0.002267,0.092781)$ \\
\hline & & & $(1.412975,0.030999,0.218746)$ & $(0.899895,0.009618,0.210720)$ \\
\hline & & & $(3.570973,0.001677,0.099605)$ & $(1.453491,0.000222,0.074069)$ \\
\hline & & & $(-0.331269,-0.005152,0.100935)$ & $(-0.711753,-0.004670,0.195593)$ \\
\hline & & \multirow[t]{3}{*}{0.8} & $(1.482429,0.032797,0.158092)$ & $(0.996478,0.009042,0.223078)$ \\
\hline & & & $(4.623204,0.002162,0.050992)$ & $(1.371019,0.000156,0.060592)$ \\
\hline & & & $(-0.092884,0.000442,0.082341)$ & $(-0.124146,0.001331,0.076157)$ \\
\hline & \multirow{5}{*}{150} & \multirow[t]{3}{*}{1.2} & $(1.194005,0.029246,0.184984)$ & $(0.783033,0.007909,0.172482)$ \\
\hline & & & $(2.407211,0.001456,0.067009)$ & $(1.033839,0.000156,0.054197)$ \\
\hline & & & $(-0.006219,-0.000185,0.049270)$ & $(-0.672792,-0.005171,0.193702)$ \\
\hline & & \multirow[t]{2}{*}{0.8} & $(1.220938,0.271811,0.112398)$ & $(0.886939,0.008259,0.218931)$ \\
\hline & & & $(2.825691,0.001249,0.026412)$ & $(1.009560,0.000079,0.058949)$ \\
\hline
\end{tabular}

TABLE 3: Number of cycles to failure.

\begin{tabular}{|c|c|c|c|c|c|c|c|c|c|c|c|c|}
\hline 15 & 20 & 38 & 42 & 61 & 76 & 86 & 98 & 121 & 146 & 149 & 157 & 175 \\
\hline 176 & 180 & 180 & 198 & 220 & 224 & 251 & 264 & 282 & 321 & 325 & 653 & - \\
\hline
\end{tabular}

TABLE 4: Waiting times (in minutes) of customers to receive service in a bank.

\begin{tabular}{lccccccccc}
\hline 0.8 & 0.8 & 1.3 & 1.5 & 1.8 & 1.9 & 1.9 & 2.1 & 2.6 & 2.7 \\
\hline 2.9 & 3.1 & 3.2 & 3.3 & 3.5 & 3.6 & 4.0 & 4.1 & 4.2 & 4.2 \\
4.3 & 4.3 & 4.4 & 4.4 & 4.6 & 4.7 & 4.7 & 4.8 & 4.9 & 4.9 \\
5.0 & 5.3 & 5.5 & 5.7 & 5.7 & 6.1 & 6.2 & 6.2 & 6.2 \\
6.7 & 6.9 & 7.1 & 7.1 & 7.1 & 7.1 & 7.4 & 7.6 & 7.7 & 6.3 \\
8.2 & 8.6 & 8.6 & 8.6 & 8.8 & 8.8 & 8.9 & 8.9 & 9.5 & 9.6 \\
9.7 & 9.8 & 10.7 & 10.9 & 11.0 & 11.0 & 11.1 & 11.2 & 11.2 & 11.5 \\
11.9 & 12.4 & 12.5 & 12.9 & 13.0 & 13.1 & 13.3 & 13.6 & 13.7 & 13.9 \\
14.1 & 15.4 & 15.4 & 17.3 & 17.3 & 18.1 & 18.2 & 18.4 & 18.9 & 19.0 \\
19.9 & 20.6 & 21.3 & 21.4 & 21.9 & 23.0 & 27.0 & 31.6 & 33.1 & 38.5 \\
\hline
\end{tabular}

TABLe 5: Number of cycles before failure of 60 electrical appliances, reported by Lawless [27].

\begin{tabular}{ccccccccccccc}
\hline 0014 & 0034 & 0059 & 0061 & 0069 & 0080 & 0123 & 0142 & 0165 & 0210 & 0381 & 0464 & 0479 \\
\hline 0556 & 0574 & 0839 & 0917 & 0969 & 0991 & 1064 & 1088 & 1091 & 1174 & 1270 & 1275 \\
1397 & 1477 & 1578 & 1649 & 1702 & 1893 & 1932 & 2001 & 2161 & 2292 & 2326 & 2337 & 2628 \\
2785 & 2811 & 2886 & 2993 & 3122 & 3248 & 3715 & 3790 & 3857 & 3912 & 4100 & 4106 & 4116 \\
4315 & 4510 & 4584 & 5267 & 5299 & 5583 & 6065 & 9701 & - & - & - & - & - \\
\hline
\end{tabular}

TABLE 6: Survival times of a group of patients suffering from head and neck cancer.

\begin{tabular}{lcccccccccccc}
\hline 12.20 & 23.56 & 23.74 & 25.87 & 31.98 & 37 & 41.35 & 47.38 & 55.46 & 58.36 & 63.47 & 68.46 & 78.26 \\
\hline 74.47 & 81.43 & 84 & 92 & 94 & 110 & 112 & 119 & 127 & 130 & 133 & 140 & 146 \\
155 & 159 & 173 & 179 & 194 & 195 & 209 & 249 & 281 & 319 & 339 & 432 & 469 \\
519 & 633 & 725 & 817 & 1776 & - & - & - & - & - & - & - & - \\
\hline
\end{tabular}




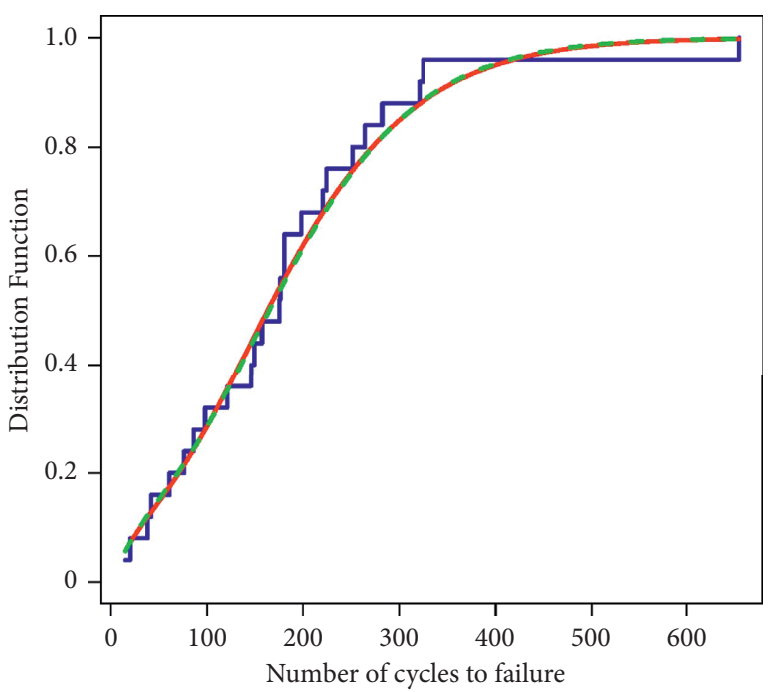

— PEL: The K-S statistic: 0.082180 , p-value $=0.995907$ --- EL: The K-S statistic: 0.087664 , p-value $=0.990698$

(a)

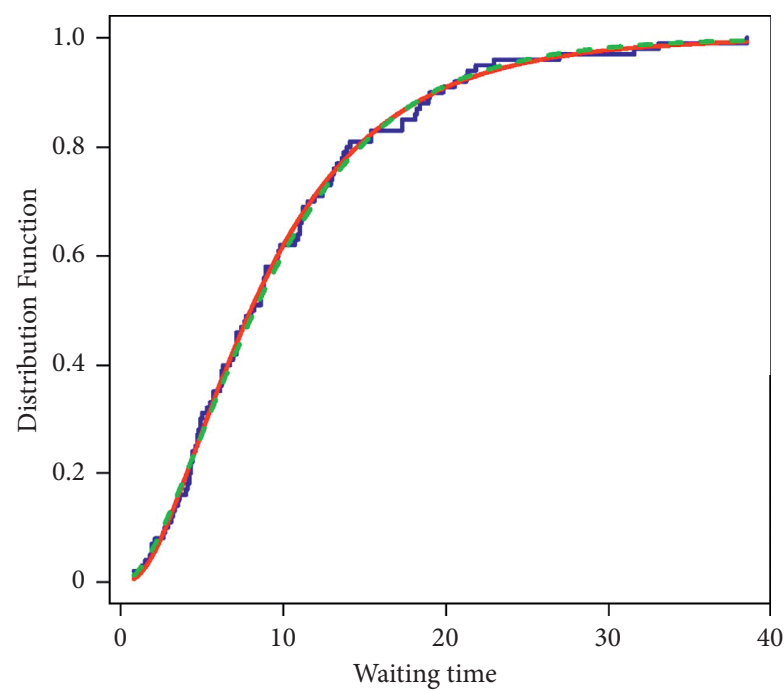

— PEL: The K-S statistic: 0.035617, p-value $=0.999579$ --- EL: The K-S statistic: 0.037998, p-value $=0.998716$

(b)

Figure 8: (a) The empirical and fitted CDF of data in Table 3. (b) The empirical and fitted CDF of data in Table 4.

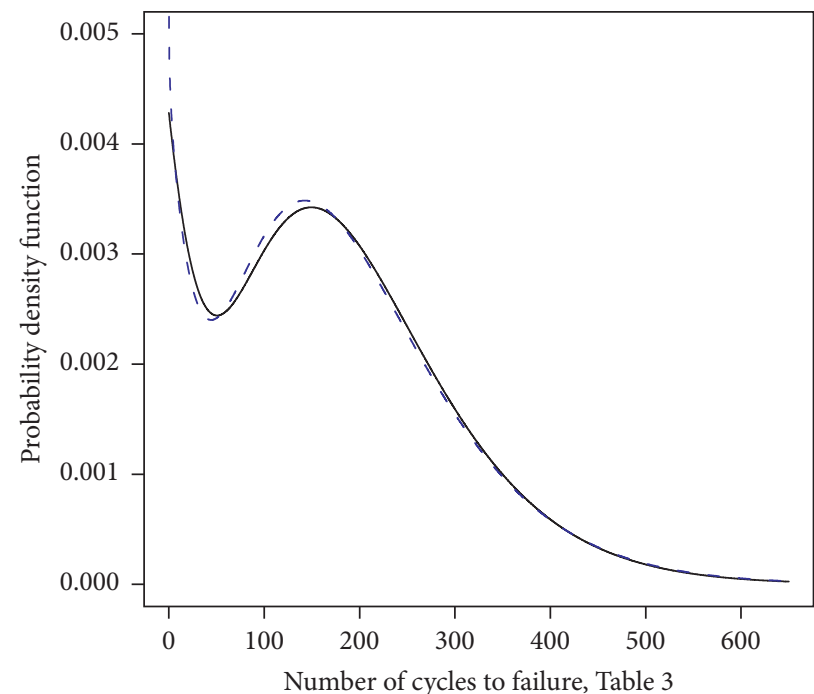

- EL: $\mathrm{k}=1, \mathrm{~m}=4, \delta=1.807, \theta=0.0185$

- - PEL: $\mathrm{k}=1, \mathrm{~m}=4, \delta=1.5846, \theta=0.0235, \gamma=0.9598$

(a)

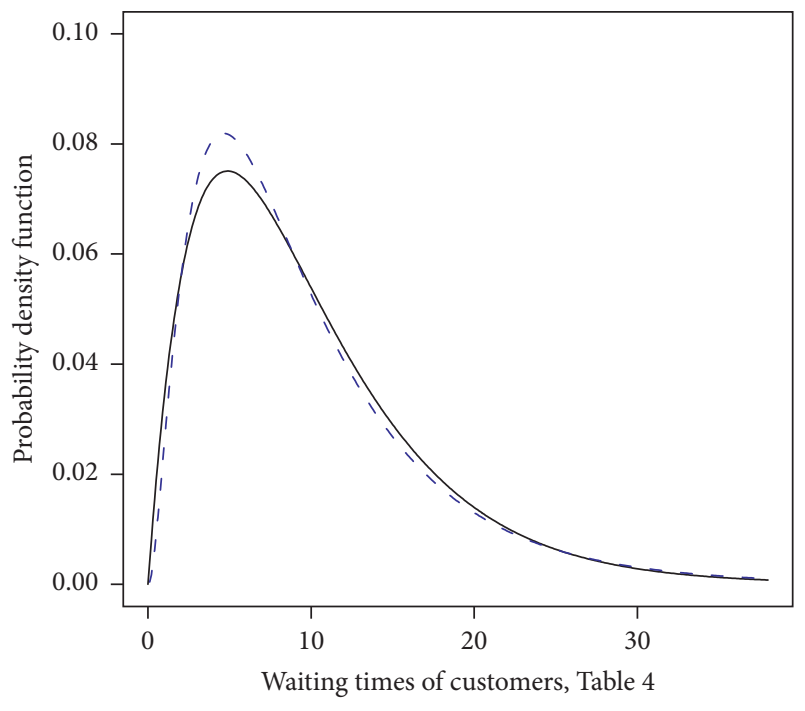

- EL: $\mathrm{k}=7, \mathrm{~m}=2, \delta=0.0051, \theta=0.2051$

- - PEL: $\mathrm{k}=9, \mathrm{~m}=6, \delta=1 \mathrm{el} 06, \theta=1.7927, \gamma=0.5545$

(b)

Figure 9: The estimated PDF of the EL and the PEL of data sets of Table 3 (a) and Table 4 (b), respectively. 


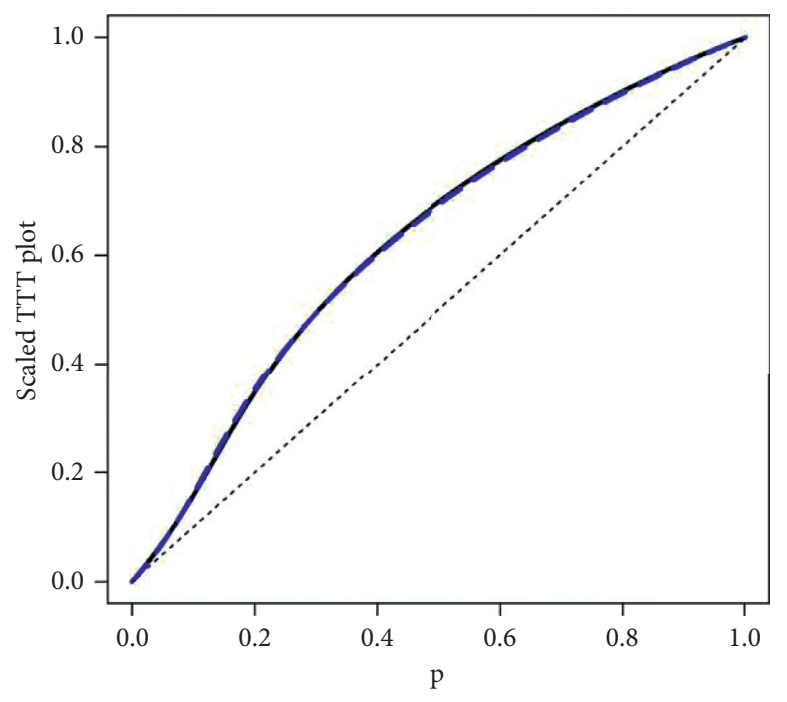

- The estimated EL for data of Table 3

--- The estimated PEL for data of Table 3

(a)

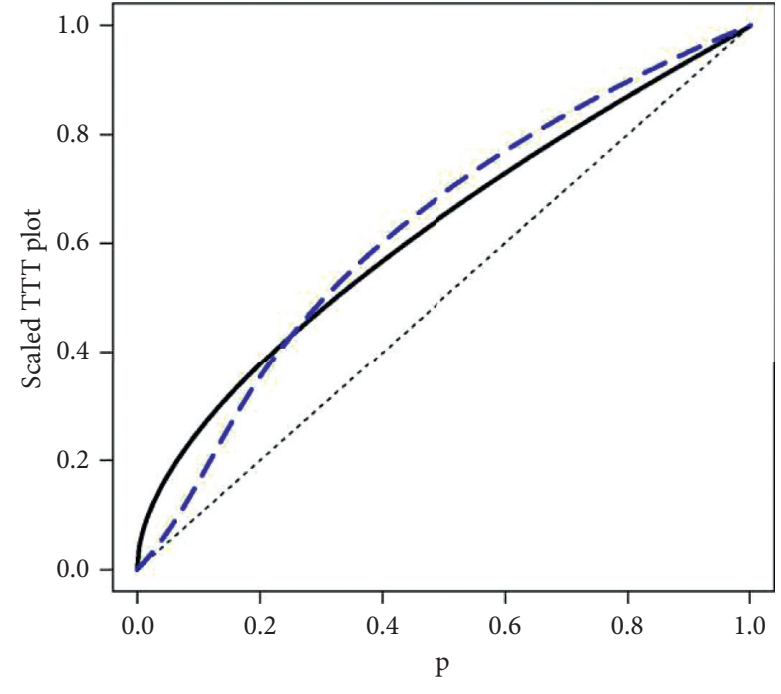

— The estimated EL for data of Table 4

--- The estimated PEL for data of Table 4

(b)

Figure 10: The estimated scaled TTT plot of the EL and the PEL of data sets of Table 3 (a) and Table 4 (b), respectively.

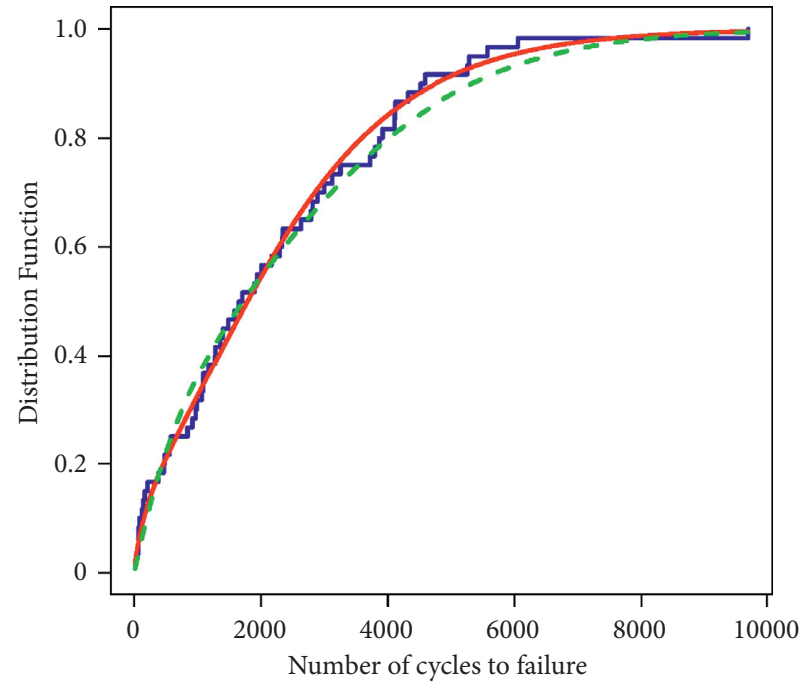

PEL: The K-S statistic: 0.064259 , p-value $=0.951852$ --- EL: The K-S statistic: 0.075124, p-value $=0.862004$

(a)

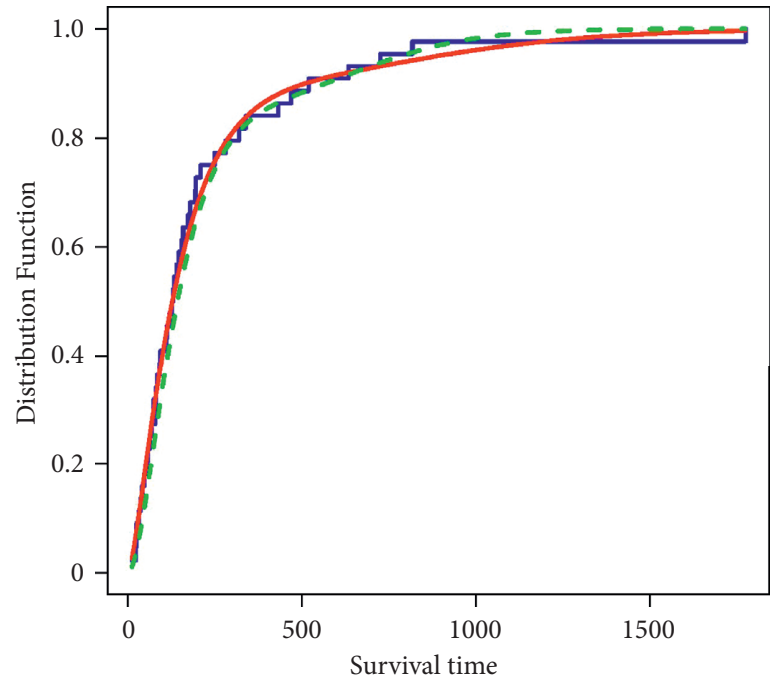

— PEL: The K-S statistic: 0.057155 , p-value $=0.997130$ -- EL: The K-S statistic: 0.092979, p-value $=0.807714$

(b)

Figure 11: (a) The empirical and fitted CDF of data in Table 5. (b) The empirical and fitted CDF of data in Table 6. 


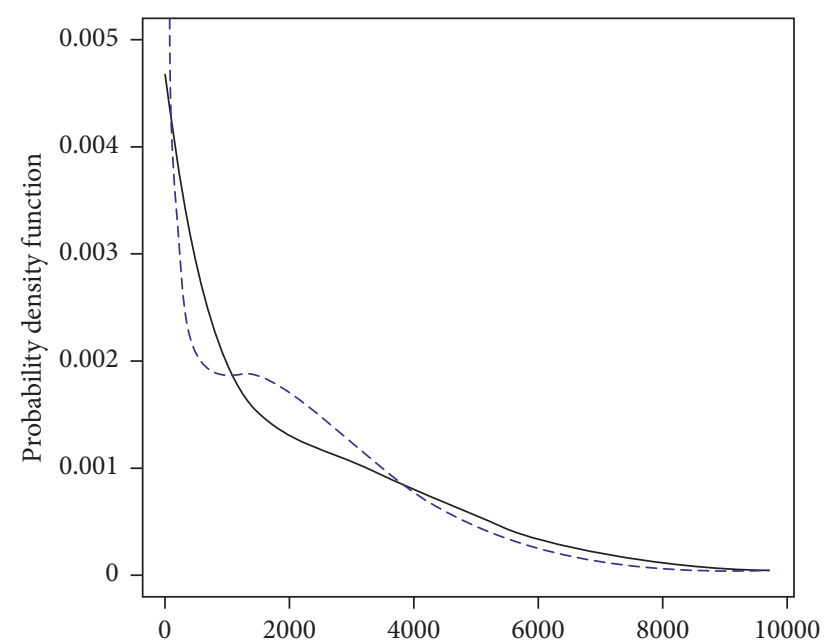

Number of cycles before failure of electrical appliances, Table 5

- EL: $\mathrm{k}=4, \mathrm{~m}=1, \delta=0.7893, \theta=0.001$

- - PEL: $\mathrm{k}=1, \mathrm{~m}=4, \delta=2.6871, \theta=0.0071, \gamma=0.7976$

(a)

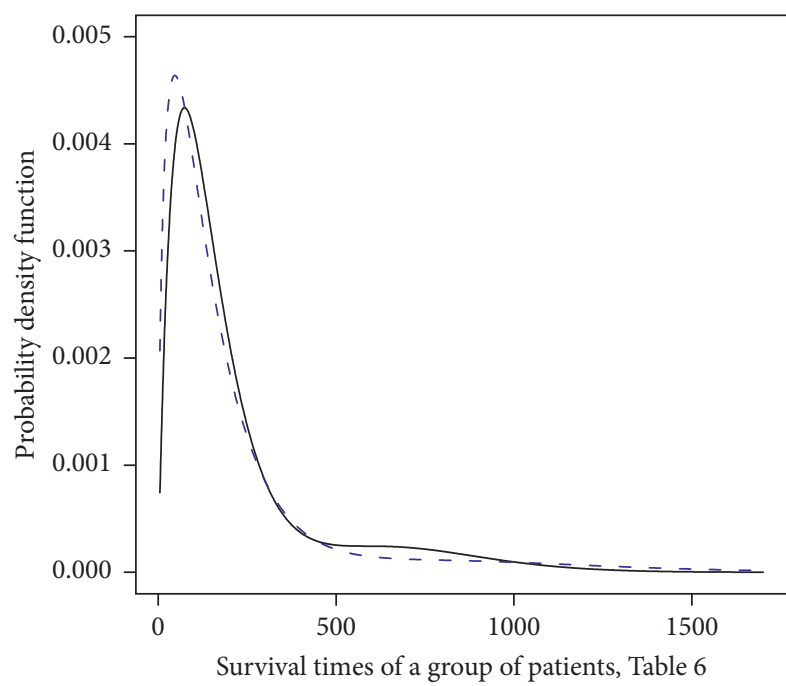

— EL: $\mathrm{k}=10, \mathrm{~m}=2, \delta=0.1451, \theta=0.0135$

- - PEL: $\mathrm{k}=10, \mathrm{~m}=2, \delta=0.0952, \theta=0.0326, \gamma=0.831$

(b)

FIgure 12: The estimated PDF of the EL and the PEL of data sets of Table 5 (a) and Table 6 (b), respectively.

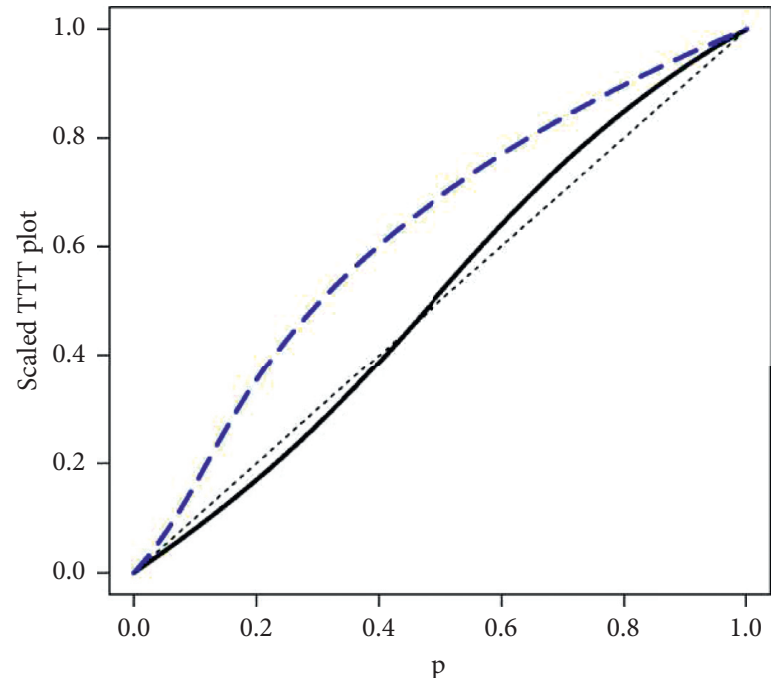

- The estimated EL for data of Table 5

--- The estimated PEL for data of Table 5

(a)

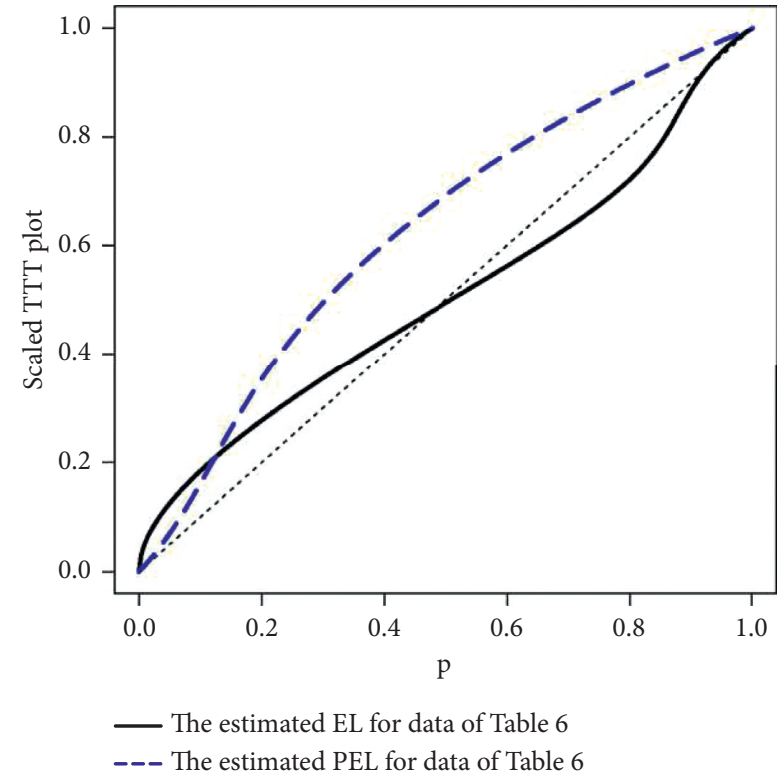

(b)

Figure 13: The estimated scaled TTT plot of the EL and the PEL of data sets of Table 5 (a) and Table 6 (b), respectively. 
TABLE 7: Results of fitting models.

\begin{tabular}{ccccccccc}
\hline Data set & Model & $k$ & $m$ & $(\widehat{\delta}, \widehat{\theta}, \widehat{\gamma})$ & AIC & K-S statistic $p$ value & CVM statistic $p$ value & AD statistic $p$ value \\
\hline & EL & 1 & 4 & $(1.8070,0.0185)$ & 309.4334 & $0.0876,0.9906$ & $0.0305,0.9766$ & $0.2805,0.9517$ \\
Table 3 & PEL & 1 & 4 & $(1.5846,0.0235,0.9598)$ & 311.4135 & $0.0822,0.9959$ & $0.0302,0.9775$ & $0.2714,0.9576$ \\
& QL & - & - & $(0.0843,0.0107)$ & 308.9154 & $0.1323,0.7736$ & $0.0542,0.8547$ & $0.3327,0.9109$ \\
& PQL & - & - & $(2.5383,0.0010,1.3536)$ & 310.9613 & $0.1101,0.9223$ & $0.0474,0.8959$ & $0.3269,0.9159$ \\
\hline \multirow{6}{*}{ Table 4} & EL & 7 & 2 & $(0.0051,0.2051)$ & 638.5809 & $0.0379,0.9987$ & $0.0236,0.9924$ & $0.1614,0.9976$ \\
& PEL & 9 & 6 & $(0.000001,1.7927,0.5545)$ & 640.2348 & $0.0356,0.9996$ & $0.0136,0.9989$ & $0.1382,0.9993$ \\
& QL & - & - & $\left(0.3 \times 10^{-6}, 0.2024\right)$ & 638.6014 & $0.0422,0.9941$ & $0.0282,0.9819$ & $0.1836,0.9943$ \\
& PQL & - & - & $(480.295,0.0304,1.4587)$ & 643.4615 & $0.0590,0.8766$ & $0.0638,0.7906$ & $0.4150,0.8333$ \\
\hline \multirow{6}{*}{ Table 5 } & EL & 4 & 1 & $(0.7893,0.001)$ & 1045.1590 & $0.0751,0.8620$ & $0.0608,0.8106$ & $0.5470,0.6987$ \\
& PEL & 1 & 4 & $(2.6871,0.0071,0.7976)$ & 1043.4850 & $0.0642,0.9518$ & $0.0271,0.9854$ & $0.2137,0.9862$ \\
& QL & - & - & $(2.4425,0.00058)$ & 1046.51 & $0.0830,0.7717$ & $0.0787,0.7010$ & $0.7385,0.5269$ \\
& PQL & - & - & $(2.8669,0.0007,0.9755)$ & 1048.525 & $0.0769,0.8430$ & $0.0892,0.6420$ & $0.7207,0.5411$ \\
\hline \multirow{2}{*}{ Table 6 } & EL & 10 & 2 & $(0.1451,0.0135)$ & 565.1387 & $0.0930,0.8077$ & $0.1054,0.5606$ & $0.6927,0.5640$ \\
& PEL & 10 & 2 & $(0.0952,0.0326,0.8310)$ & 562.9648 & $0.0571,0.9971$ & $0.0172,0.9990$ & $0.1515,0.9985$ \\
& QL & - & - & $(761.09,0.0044)$ & 568.0193 & $0.1454,0.2816$ & $0.1777,0.3159$ & $0.9673,0.3744$ \\
& PQL & - & - & $(565.2588,0.00637,0.9407)$ & 569.6834 & $0.1310,0.4023$ & $0.1438,0.4100$ & $0.8765,0.4283$ \\
\hline
\end{tabular}

\section{Conclusions}

Lindley distribution and its generalizations are useful in reliability theory and for analyzing real data sets. Here, we introduce one new scale-invariant generalization of the Lindley distribution and its power generalization and study their traits and the problem of estimation of their parameters. The models are not very complicated but accommodate different and useful forms of hazard rate function (MRL or median residual life functions). The simulation results show that MLE is applicable and efficient. Four real data sets have been analyzed, and the results show that the proposed models can fit them conveniently.

\section{Data Availability}

The lifetime data used to support the findings of this study are included within the article.

\section{Conflicts of Interest}

The authors declare that there are no conflicts of interest.

\section{Acknowledgments}

Mohamed Kayid acknowledges financial support from the Researchers Supporting Project number (RSP-2021/392) King Saud University, Riyadh, Saudi Arabia.

\section{References}

[1] D. V. Lindley, "Fiducial distributions and Bayes' theorem," Journal of the Royal Statistical Society: Series B (Methodogical), vol. 20, no. 1, pp. 102-107, 1958.

[2] M. E. Ghitany, B. Atieh, and S. Nadarajah, "Lindley distribution and its application," Mathematics and Computers in Simulation, vol. 78, no. 4, pp. 493-506, 2008.

[3] R. Shanker and A. Mishra, "A Quasi Lindley distribution," African Journal of Mathematics and Computer Science Research, vol. 6, no. 4, pp. 64-71, 2013.
[4] R. Shanker and A. H. Ghebretsadik, "A new Quasi Lindley distribution," International Journal of Statistics and Systems, vol. 8, pp. 143-156, 2013.

[5] F. Merovci and V. K. Sharma, "The Beta-lindley distribution: properties and applications," Journal of Applied Mathematics, vol. 2014, Article ID 198951, 2014.

[6] H. Zakerzadeh and A. Dolati, "Generalized Lindley distribution," Journal of Mathematical Extension, vol. 3, pp. 13-25, 2009.

[7] E. Ibrahim, F. Merovci, and M. Elgarhy, "A new generalized Lindley distribution," Mathematical Theory and Modeling, vol. 3, pp. 30-47, 2013.

[8] R. Shanker, K. K. Shukla, R. Shanker, and T. A. Leonida, "A three-parameter Lindley distribution," American Journal of Mathematics and Statistics, vol. 7, no. 1, pp. 15-26, 2017.

[9] M. Sankaran, "The discrete Poisson-lindley distribution," Biometrics, vol. 26, no. 1, pp. 145-149, 1970.

[10] H. Zamani and N. Ismail, "Negative binomial-lindley distribution and its application," Journal of Mathematics and Statistics, vol. 6, no. 1, pp. 4-9, 2010.

[11] M. E. Ghitany, D. K. Al-Mutairi, N. Balakrishnan, and L. J. AlEnezi, "Power Lindley distribution and associated inference," Computational Statistics \& Data Analysis, vol. 64, pp. 20-33, 2013.

[12] D. K. Al-Mutairi, M. E. Ghitany, and D. Kundu, "Inferences on stress-strength reliability from Lindley distributions," Communications in Statistics-Theory and Methods, vol. 42, no. 8, pp. 1443-1463, 2013.

[13] A. A. Al-Babtain, H. A. Eid, N. A. A-Hadi, and F. Merovci, "The five parameter Lindley distribution," Pakistan Journal of Statistics, vol. 31, no. 4, pp. 363-384, 2014.

[14] M. E. Ghitany, D. K. Al-Mutairi, and S. M. Aboukhamseen, "Estimation of the reliability of a stress-strength system from power Lindley distributions," Communications in Statistics-Simulation and Computation, vol. 44, no. 1, pp. 118-136, 2015.

[15] D. K. Al-Mutairi, M. E. Ghitany, and D. Kundu, "Inferences on stress-strength reliability from weighted Lindley distributions," Communications in Statistics-Theory and Methods, vol. 44, no. 19, pp. 4096-4113, 2015.

[16] A. M. Abouammoh, A. M. Alshangiti, and I. E. Ragab, "A new generalized Lindley distribution," Journal of Statistical 
Computation and Simulation, vol. 85, no. 18, pp. 3662-3678, 2015.

[17] M. Ibrahim, A. S. Yadav, H. M. Yousof, H. Goual, and G. G. Hamedani, "A new extension of Lindley distribution: modified validation test, characterizations and different methods of estimation," Communications for Statistical Applications and Methods, vol. 26, no. 5, pp. 473-495, 2019.

[18] P. Marthin and G. S. Rao, "Generalized Weibull-Lindley (GWL) distribution in modeling lifetime data," Journal of Mathematics, vol. 2020, Article ID 2049501, 15 pages, 2020.

[19] A. A. Al-Babtain, A. H. N. Ahmed, and A. Z. Afify, "A new discrete analog of the continuous Lindley distribution, with reliability applications," Entropy, vol. 22, no. 6, p. 603, 2020.

[20] R. K. Joshi and V. Kumar, "Lindley Gompertz distribution with properties and applications," International Journal of Statistics and Applied Mathematics, vol. 5, no. 6, pp. 28-37, 2020.

[21] A. Z. Afify, M. Nassar, G. M. Cordeiro, and D. Kumar, "The Weibull Marshall-Olkin Lindley distribution: properties and estimation," Journal of Taibah University for Science, vol. 14, no. 1, pp. 192-204, 2020.

[22] C. Chesneau, L. Tomy, J. Gillariose, and F. Jamal, "The inverted modified Lindley distribution," Journal of Statistical Theory and Practice, vol. 14, no. 46, 2020.

[23] A. Algarni, "On a new generalized Lindley distribution: properties, estimation and applications," PLoS One, vol. 16, no. 2, 2021.

[24] R. E. Glaser, "Bathtub and related failure rate characterizations," Journal of the American Statistical Association, vol. 75, no. 371, pp. 667-672, 1980.

[25] C. D. Lai and M. Xie, Stochastic Aging and Dependence for Reliability, Springer, New York, NY, USA, 2006.

[26] J. Mi, "Bathtub failure rate and upside-down bathtub mean residual life," IEEE Transactions on Reliability, vol. 44, no. 3, pp. 388-391, 1995.

[27] J. F. Lawless, Statistical Models and Methods for Lifetime Data, John Wiley and Sons, Inc., Hoboken, NJ, USA, 2003.

[28] R. Shanker, "On generalized lindley distribution and its applications to model lifetime data from Biomedical science and engineering," Insights in Biomed, vol. 1, no. 2, 2016.

[29] B. Efron, "Logistic regression, survival analysis, and the Kaplan-Meier curve," Journal of the American Statistical Association, vol. 83, no. 402, pp. 414-425, 1988. 